[Accepted for Publication in Science in Context]

\title{
The Strong and Weak Senses of Theory-Ladenness of Experimentation: Theory-Driven versus Exploratory Experiments in the History of High-Energy Particle Physics
}

\author{
Koray Karaca \\ University of Wuppertal \\ Interdisciplinary Centre for Science and Technology Studies (IZWT) \\ University of Wuppertal \\ Gaußstr. 20 \\ 42119 Wuppertal, Germany
}

karacak@gmail.com

\begin{abstract}
Argument
In the theory-dominated view of scientific experimentation, all relations of theory and experiment are taken on a par; namely, that experiments are performed solely to ascertain the conclusions of scientific theories. As a result, different aspects of experimentation and of the relation of theory to experiment remain undifferentiated. This in turn fosters a notion of theoryladenness of experimentation (TLE) that is too coarse-grained to accurately describe the relations of theory and experiment in scientific practice. By contrast, in this article, I suggest that TLE should be understood as an umbrella concept that has different senses. To this end, I introduce a three-fold distinction among the theories of high-energy particle physics (HEP) as background theories, model theories and phenomenological models. Drawing on this categorization, I contrast two types of experimentation, namely, "theory-driven" and "exploratory" experiments, and I distinguish between the "weak" and "strong" senses of TLE in the context of scattering experiments from the history of HEP. This distinction enables to identify the exploratory character of the deep-inelastic electron-proton scattering experimentsperformed at the Stanford Linear Accelerator Center (SLAC) between the years 1967 and 1973 - thereby shedding light on a crucial phase of the history of HEP, namely, the discovery of "scaling", which was the decisive step towards the construction of quantum chromo-dynamics (QCD) as a gauge theory of strong interactions.
\end{abstract}

\section{Introduction}

The debate concerning the relationship between theory and experiment has a long history in the literature of philosophy of science. The reason why this debate has been so long-lived can be traced to its philosophical implications that pertain to epistemological issues such as theorytesting and theory-construction, as well as to perennial debates such as the realism-empiricism debate. During the first three quarters of the twentieth century, the scientific methodologies changed radically, whereas the conception of scientific experimentation remained essentially unchanged. Pierre Duhem was the first to scrutinize the relation between theory and experiment in his The Aim and Structure of Scientific Theory (Duhem1954). The different roles Duhem 
ascribed to experimentation are ones that are subordinated to scientific theorizing. Duhem recognized only two types of experimentation; namely, "experiment of application" and "experiment of testing". In Duhem's view, an experiment of application "does not aim at discovering whether accepted theories are accurate or not; it merely intends to draw on these theories" (Ibid, 183). In contrast, an experiment of testing is intended to show whether a particular prediction holds true or not. However, he famously argued that the prediction of a particular phenomenon requires a whole theoretical group and that no experiment can refute an isolated hypothesis:

The only thing the experiment teaches us is that among the propositions used to predict the phenomenon and to establish whether it would be produced, there is at least one error; but where this error lies is just what it does not tell us. (Ibid., 185)

Duhem's theory-dominated conception of scientific experimentation survived in the logical positivist tradition; even though, in sharp contrast to Duhem's holism, the logical positivist philosophy of science acknowledged experimentation as one of the conventional ways of theory testing in accordance with their method of verification. By contrast, in his Logic of Scientific Discovery, Karl Popper rejected verificationism and instead defended falsificationism as the proper methodological framework for the appraisal of scientific theories (Popper, 1959). Even though there existed a clear distinction between Popper's view of scientific method and that of logical positivists, this distinction did not bring about any essential difference between their standpoints concerning the role of experimentation in scientific practice. Like logical positivists, Popper subordinated experimentation to theorizing and acknowledged its purpose as being guided by theoretical commitments alone. In Popper's view:

The theoretician puts certain definite questions to the experimenter, and the latter, by his experiments, tries to elicit a decisive answer to these questions, and to no others [...] Theory dominates the experimental work from its initial planning up to the finishing touches in the laboratory. (Ibid., 89)

During the post-positivist era, the theory-dominated conception of scientific experimentation almost remained unchanged; but it was used as an argument to defend relativism in science against its positivist conceptions. During this period, the emphasis was mainly given to the relationships between theoretical commitments and sense perception. After its suggestion by Norwood Russell Hanson in his Patterns of Scientific Discovery, the thesis of theory-ladenness of observation ${ }^{1}$ became a slogan under which the dominance of theory-or more generally, of theoretical concepts — over sense experience was highlighted (Hanson 1960). ${ }^{2}$ One natural extension of the thesis of theory-ladenness of observation to experimentation has been the thesis of theory-ladenness of experimentation (TLE). In his The Structure of Scientific Revolutions, Kuhn construed TLE as "paradigm-ladenness" and argued that experimentation is always guided by theoretical commitments that belong to the paradigm of a particular science (Kuhn 1970).

\footnotetext{
${ }^{1}$ For a discussion of the debate on theory-ladenness of observation, see, e.g., Radder 2006, chap. 3.

${ }^{2}$ Paul Feyerabend, who defended a radical version of relativism in science, criticized the thesis of theory-ladenness of observation by arguing that "observations (observation terms) are not merely theory-laden ... but fully theoretical (observation statements have no "observational core")" (Feyerabend 1981, 10).
} 
Until the early 1980s, the philosophy of experimentation had been framed by TLE, and this theory-dominated view had been largely accepted as the standard view of scientific experimentation; according to which experimentation is always guided by well-defined theoretical accounts and is solely intended to ascertain the conclusions of those accounts. Ian Hacking's rejection of the standard view in the early 1980s opened the door to a new conception of scientific experimentation. In his Representing and Intervening, Hacking argued that it would be wrong to subordinate experiment to theory (Hacking 1983). Unlike the proponents of the standard view, Hacking conceived of experimentation as being by itself capable of producing genuine knowledge of the world. Hacking's conception of scientific experimentation started a new movement in philosophy of science, often referred to as New Experimentalism ${ }^{3}$, which suggested that experimentation should be studied for its own sake, and not merely as subordinate to scientific theorizing. ${ }^{4}$

It is to be noted that Hacking's discussion of the relationship between theory and experiment in some parts of Representing and Intervening is ambiguous. While he admits that he "make[s] no claim that experimental work could exist independently of theory," he claims that "much truly fundamental research precedes any relevant theory whatsoever" (Ibid., 158). In a later work, Hacking seems to be much more precise and makes a three-fold distinction among theories used in experimentation (Hacking 1992). According to this distinction, what Hacking calls "systematic theories" are high-level theories and do not necessarily have experimental consequences on their own. Whereas what he calls "topical hypotheses" are relatively low-level empirical generalizations and serve to connect the systematic theory to the phenomena of interest. And, as a third category, Hacking states what he calls "background theories" that concern the design and the workings of the apparatus and instruments used during the course of experimentation.

In the vein of Hacking's famous dictum that "[e]xperimentation has many lives of its own" (Hacking 1983, 165), Peter Galison rejected any reductive hierarchy between theory and experiment, and he instead offered a model of "intercalated periodization" that characterizes the relationships between theorizing, experimentation and instrumentation as exhibiting diverse levels of interaction each of which has its own "partial autonomy" (Galison 1987). Moreover, what is more interesting for the purpose of the present paper is the distinction Galison draws between what he calls long, medium and short term constraints of experimental inquiry (Ibid., chap. 5). According to this distinction, for example, the idea of unification of fundamental forces of nature constitutes a long-term constraint for the experimental activities in the field of particle physics. However, gauge theories and different models and phenomenological laws used in particle physics constitute respectively medium and short-term constraints for the same field (Ibid., 254).

The above discussion indicates that even though both Hacking and Galison draw various distinctions between different kinds of theoretical commitments involved in experimentation, they do not deal with the philosophical implications of this categorization for the notion of TLE. If experimentation involves various kinds of theoretical commitments, then it is epistemologically of interest to ask whether this brings about varying senses of TLE. In this paper, I shall address this issue in the context of experiments from the history of HEP.

\footnotetext{
${ }^{3}$ For a review, see, e.g., Ackermann 1989.

${ }^{4}$ See, most notably, Franklin 1986, 1990; Galison 1987; Giere 1988; and Gooding 1990.
} 


\section{Recent Perspectives in the Philosophy of Scientific Experimentation}

The theory-dominated view of scientific experimentation suggests that experiments are performed solely to ascertain the conclusions of scientific theories. Therefore, in this view, different aspects of experimentation and of the relation of theory to experiment remain undifferentiated. This in turn fosters a conception of TLE that is too coarse-grained to accurately describe the scientific practice. In more recent years, however, the relation between theory and experiment has been examined in somewhat more detail with regard to TLE. Friedrich Steinle argued that the conception of TLE as conceived in the theory-dominated view falls short in capturing both the complexity and diversity of scientific experimentation (Steinle 1997, 2002). Steinle reaches this conclusion in the light of a contrast he introduces between two types of experimentation, namely, "theory-driven" and "exploratory" experiments. ${ }^{5}$ In his view, theorydriven experimentation exhibits features that conform to the standard view of scientific experimentation; namely, it is "done with a well-formed theory in mind, from the very-first idea, via the specific design and the execution, to the evaluation" (Steinle 1997, 69). Theory-driven experiments can also serve other purposes, such as the determination of a numerical parameter, or the use of theories as a heuristic tool within the search for a new effect (Ibid., 69-70). By contrast, what Steinle calls exploratory experimentation "typically takes place in those periods of scientific development in which - for whatever reasons - no well-formed theory or even no conceptual framework is available or regarded as reliable" (Ibid., 70). However, in Steinle's view, "despite its independence from specific theories, the experimental activity [in exploratory experimentation] may well be highly systematic and driven by typical guidelines" (Ibid., 70). Steinle's paradigmatic examples of exploratory experimentation are from the early history of electromagnetism and include experiments on static electricity performed by Charles Dufay, André-Marie Ampère and Michael Faraday. Here, it is to be noted that what Steinle means by "new research field" is a research domain in which there yet exists no well-defined and wellestablished theoretical framework.

According to Steinle, "[e]xploratory experimentation is not one specific and well-defined procedure, but includes a whole bundle of different experimental strategies" (Ibid., 73). Steinle identifies some of those exploratory strategies as follows:

- Varying a large number of different experimental parameters;

- determining which of the different experimental conditions are indispensable, which are only modifying;

- looking for stable empirical rules;

- finding appropriate representations by means of which those rules can be formulated;

- forming experimental arrangements which involve only the indispensable conditions, thus presenting the rule in particular clarity. (Ibid., 70)

Moreover, Steinle argues that the findings of exploratory experiments might have significant implications on our understanding of existing theoretical concepts, in that the attempt

\footnotetext{
${ }^{5}$ At around the same time as Steinle, the notion of "exploratory experimentation" was also used by Richard Burian (Burian 1997) and earlier by Rose-Mary Sargent (Sargent 1995). More recently, exploratory experimentation has received more attention from philosophers of science; see, e.g., Franklin 2005; Burian 2007; Elliott 2007; Marcum 2007; O’Malley 2007; Waters 2007; and Cobb 2009.
} 
to formulate the regularities suggested by exploratory experiments might require "the revision of existing concepts and categories, and the formation of new ones" (Ibid., 70).

Steinle's work has triggered a whole new debate centering on the possibility of theoryfree experimentation. Michael Heidelberger has distinguished between different levels of experimentation, namely, that "experimentation at the causal level, where instrumental manipulation is distinguished, and experimentation taking place at the theoretical level, where the results at the causal level are represented in a theoretical structure" (Heidelberger 2003, 145). Heidelberger has argued that in domains occupied by well-established theories the two levels of experimentation are inextricably connected to each other, and thus experimentation proceeds at both levels. However, argues Heidelberger, if a new domain is explored, experimentation proceeds only at the causal level and is theory-free. As the foregoing discussion indicates, there exist parallels between the views of Heidelberger and Steinle on scientific experimentation; however Steinle does not argue against TLE and does not seem to deny the role of background theories in the case of exploratory experiments. As a result, unlike Heidelberger, he does not suggest the possibility of theory-free experimentation.

In contrast to Heidelberger, Martin Carrier has argued that "performing experiments and measuring physical quantities rely on overarching observation theories" (Carrier 1998, 182). Carrier has distinguished observation theories that directly enter experimental processes from what he has called explanation theories that just account for the phenomena under consideration. According to Carrier's distinction, the difference between observation and explanation theories is not of a fundamental nature, but rather concerns a mode of usage. That is, the experimental context determines which theory is to be used as an observation theory and which one as an explanation theory. While a theory is used as an observation theory in a certain experimental context, it can as well be used as an explanation theory in another context. This in turn means that observation theories can be as high-level and systematic as explanation theories. Note that this view of Carrier's clearly stands at odds with that of Hacking, who maintains that systematic high-level theories typically do not play substantive role in experimentation.

In contrast to the possibility of theory-free experimentation, Hans Radder has argued that "materially realizing a stable correlation and knowing what can be learned about the objects from inspecting the apparatus depends upon theoretical insights about the experimental system and its environment" (Radder 2003, 165). In Radder's view, the establishment of a stable correlation between some feature of the phenomena being examined and some feature of the experimental set up is always constitutive of scientific experimentation. Unlike Carrier, however, Radder attributes a broader role to theory in scientific experimentation by arguing that "the immediate or later significance of experiments is affected by the theoretical context in which they are situated" (Ibid., 163).

The preceding discussion indicates that a significant portion of the recent literature on scientific experimentation has been devoted to the possibility of theory-free experimentation. However, what is more interesting for the aim of the present paper are the parallels between the approaches of Hacking, Carrier and Radder. Notwithstanding the existing differences between their accounts, they all make distinctions among theories involved in experimental processes. While Hacking makes distinctions among what he calls systematic theories, topical hypotheses and theories of instrumentation, Carrier distinguishes between observation and explanation 
theories. And, Radder distinguishes theories used in instrumentation from those used in interpreting experimental findings. ${ }^{6}$

In this paper, I shall follow an approach similar to those of the above-mentioned studies. In the next section, I shall categorize the theories of HEP into three main types: namely, background theory, model theory and phenomenological model. I shall argue that each category here bears a different set of relationships to experimentation and thus gives rise to a different set of theoretical commitments in experimental research in HEP. In the ensuing sections of the present paper, I shall explore the philosophical implications of the above categorization of the theories of HEP for the notion of TLE. To this end, in contrast to the undifferentiated notion of TLE underlying the theory-dominated view of scientific experimentation, I shall characterize and distinguish between the "weak" and "strong" senses of the notion of TLE. I shall use the term "theory-drivenness of experimentation" (TDE) to denote the form of theory-ladenness underlying the theory-driven type of experimentation. In the same spirit as Steinle, I shall characterize theory-driven experimentation as a specific type of experimentation that is performed under the continuous guidance of some theoretical account typically with the aim to ascertain the conclusions of the same account. That is to say, in theory-driven type of experiments, all successive stages of experimentation, from planning and design up to data analysis and data-interpretation, are performed by appealing to the theoretical accounts of the target phenomena. By contrast, I shall characterize the weak sense as theory-ladenness in experimentation due to the utilization of theoretical considerations that have no guiding power on the progress of the experimental process.

\section{A Three-Fold Distinction in Theorizing in HEP: Background Theories, Model Theories and Phenomenological Models}

The distinction between what are called "background theories" and "phenomenological models" of science is held for a quite long time by philosophers of science — most prominently by Nancy Cartwright, Margaret Morrison and Mary Morgan. In her How the Laws of Physics Lie, Cartwright has famously argued that the description of natural phenomena is generally provided not by our "fundamental theories", but rather by phenomenological models obtained from fundamental laws through approximations and emendations that are not dictated by those fundamental laws (Cartwright 1983). In a later work, Cartwright and her collaborators have argued that the methods and aims of phenomenological model building enjoy a great deal of independence from fundamental theory (Cartwright, Shomar and Suarez 1995). In a similar vein, Morgan and Morrison have stressed the importance of and the need for model building in scientific practice and argued that in many cases the explanation and prediction of the particular features of natural phenomena are provided by models that are constructed by supplementing the fundamental theory by further structural and conceptual elements outside of its theoretical framework (Morgan and Morrison 1999). On this basis, they have argued that models embody an element of independence from both theory and data. Morrison has given careful consideration to phenomenological model building and argued that phenomenological models function independently of theory, and yet this independence is only partial; because the construction of

\footnotetext{
${ }^{6}$ A similar distinction was drawn by Giora Hon, who offered an account of experimental error. In an attempt to identify possible sources of error in experimental process, Hon draws a distinction between what he calls background theories and moral theories; while the former are necessary for the instrumentation in experiments, the latter are necessary for the interpretation of the outcomes of experiments (Hon 2003).
} 
phenomenological models and their applicability to natural phenomena can be reliant on some important features of the fundamental theory (Morrison 1999). ${ }^{7}$ In what follows, I shall take my cue from the above discussion and draw a three-fold distinction among the theories of HEP.

HEP is primarily concerned with the fundamental constituents of matter and their mutual interactions. In nature, there are known to exist four fundamental interactions (or forces): namely, electromagnetic, weak, strong and gravitational forces. The electromagnetic force is a long-range force and acts between electrically charged particles and photons. The weak force is a short-range force and acts between all types of elementary particles. The strong force is also a short-range force and acts between particles called "hadrons", e.g., nucleons (protons or neutrons), and "quarks" which are believed to constitute hadrons. ${ }^{8}$ Since the magnitude of gravitational force is very weak as compared to those of other types of fundamental forces, any possible gravitational effects are typically neglected in HEP calculations. Most of the interactions between sub-atomic particles do not occur under normal circumstances in nature; but they are created and detected during very energetic collisions, as are done in particle accelerators. Since the quantum mechanical effects dominate over the classical ones at subatomic domains, the interactions between sub-atomic particles are properly treated by various theories and models that conform to the basic postulates of the quantum mechanics (QM). ${ }^{9}$ In HEP, what I shall call "background theories" are those theories that provide the basic rules and principles that govern the major aspects of fundamental forces. However, since fundamental forces exhibit features that are quite distinct from each other, the rules and principles provided by background theories for the description of fundamental forces fall short in explaining and predicting the interaction-specific features of sub-atomic phenomena. ${ }^{10}$ In HEP, what I shall call a "model theory" is a theory that is constructed under the theoretical framework of a background theory for the specific purpose of explaining the interaction-specific features of fundamental force fields. As compared to background theories, model theories can be said to be more elaborate, in that their theoretical frameworks accommodate a relatively greater number of conceptual and structural elements that involve interaction-specific principles, hypotheses and mechanisms that background theories do not typically possess. By virtue of these structural and conceptual elements, model theories are able to relate to their target phenomena and provide explanations as well as experimentally testable predictions about sub-atomic phenomena. In the ensuing discussion, I shall illustrate the distinction of background and model theories by considering quantum field theory (QFT) in relation to gauge theories of particle physics; namely, quantum electrodynamics (QED), electro-weak theory (EWT) and quantum chromo-dynamics (QCD).

QFT is the underlying theory of all types of interactions between sub-atomic particles. It is a relativistic field theory that provides rules - such as "canonical quantization" - to quantize fundamental interactions, as well as mathematical techniques - such as "renormalization"- to extract experimentally ascertainable quantities — such as binding energy and scattering cross

\footnotetext{
${ }^{7}$ Note that Michael Redhead (Redhead 1980a) and Stephan Hartmann (Hartman 1998, 1999) proposed similar distinctions in the context of QFT.

${ }^{8}$ In present-day HEP, the term "hadron" refers to a subatomic particle that is subject to strong interaction. Hadrons are not considered to be fundamental particles but composed of quarks, antiquarks, and gluons. And, the strong nuclear force, according to our current understanding of fundamental interactions, represents the interactions between quarks and gluons as detailed by the theory of QCD.

${ }^{9}$ For a philosophical introduction to QM, see, e.g., Healey 1989; Hughes 1992; and Albert 1994.

${ }^{10}$ Here, the term "interaction" signifies any of the fundamental interactions of nature previously mentioned.
} 
section-from theoretical calculations. ${ }^{11}$ Each aforementioned gauge theory is a specific quantum field theory that uses the formalism of QFT and that applies to a particular type of interaction. While QED concerns electromagnetic interactions between electrically charged particles and photons, QCD concerns strong interactions; and EWT provides a unified description of weak and electromagnetic forces. The above discussion suggests that, in HEP, even though all fundamental force fields are treated as being quantized fields in accordance with QFT, the interaction specific features of fundamental forces are accounted for by different quantum field theories.

In QFT, the Lagrangian density function (or simply the Lagrangian) is taken to be the basic dynamical quantity that is used to calculate the field equations governing the dynamics of the fundamental forces and their associated physical quantities such as energy and momentum values. Since QFT does not single out any specific type of fundamental interaction, it does not impose any specific mathematical form for the Lagrangian. ${ }^{12}$ Rather, it provides the mathematical formalism necessary for a dynamical analysis of fundamental forces. Given an interaction-specific Lagrangian, QFT provides rules and techniques as to how to carry out theoretical calculations as well as to extract experimentally ascertainable consequences from these calculations. Whereas, in QED, EWT and QCD, the exact mathematical form of the Lagrangian is fixed. Since these theories describe different types of interactions, they adopt Lagrangians of different mathematical forms for the explanation of their target phenomena. In addition, these theories possess different symmetries; while QED and EWT exhibit respectively $\mathrm{U}(1)$ and $\mathrm{SU}(2) \otimes \mathrm{U}(1)$ gauge symmetries, QCD exhibits $\mathrm{SU}(3)$ gauge symmetry. In each of these theories, the underlying symmetry principle is represented as the invariance of the Lagrangian under a symmetry transformation. It is to be noted that neither of these symmetries is dictated by QFT itself. Rather, in each case, the incorporation of the symmetry formalism into the theoretical structure of the theory is guided by an additional symmetry argument stating the conservation of a particular physical quantity during the mediation of the interaction under consideration. In QFT, the term "conserved current" signifies the conservation of a physical quantity during the course of an interaction; thereby indicating the existence of a particular symmetry principle. For instance, in QED, the electromagnetic current and the electric charge respectively represent the conserved current and the associated conserved quantity. Similarly, in EWT and QCD, the conserved quantities are respectively "weak-isospin" and "color charge". 13

Moreover, both EWT and QCD explain their target phenomena by appealing to certain specialized mechanisms that are again not dictated by QFT itself. In EWT, the way how the intermediate vector bosons-i.e., the mediators of the weak force-acquire their mass is explained by a mechanism called the "Higgs Mechanism". ${ }^{14}$ And, in QCD, the motions of quarks within the nucleons are accounted for through a mechanism called "asymptotic freedom." 15 It is to be noted that these mechanisms are not directly derivable from QFT. Rather, their construction and incorporation into the theoretical structure of their corresponding theories require further theoretical considerations that are not readily available in the theoretical framework of QFT.

\footnotetext{
${ }^{11}$ For a philosophical discussion of central issues in QFT, as well as more generally in particle physics, see, e.g., Redhead 1980b, 1982; Brown and Harré 1988; Auyang 1995; Teller 1995; Clifton 1996; Cao 1997, 1999;

Falkenburg 2007; and Ruetsche 2011.

${ }^{12}$ Lagrangian is a mathematical function that is defined as the difference between the kinetic and potential energies of a dynamical system.

${ }^{13}$ For these concepts, see, e.g., Griffiths 2008.

${ }^{14}$ For this mechanism, see, e.g., Griffiths 2008, sec.10.9. For a historical account, see Karaca forthcoming.

${ }^{15}$ See Griffiths 2008, sec. 8.6.
} 
My discussion so far has suggested that even though the construction of QED, EWT and QCD largely depends upon QFT for the quantization of force fields, their theoretical frameworks accommodate interaction-specific conceptual elements in the form of hypotheses - such as symmetry principles - and mechanisms that QFT does not readily offer. And, it is by virtue of these structural elements that these theories are capable of accounting for the dynamics of fundamental forces. Therefore, I categorize QED, EWT and QCD as interaction-specific quantum field theories and call them "model theories" of QFT.

An important inadequacy of the model theories of HEP is that even though they account for the very essential features of their target phenomena, they fail to capture their various underlying complexities. As a result, experiments in HEP are typically conducted by appealing to those models that are often referred to as "phenomenological models." ${ }^{16}$ Just as model theories are constructed by supplementing background theories of HEP to explain the basic features of elementary particles and to understand their mutual interactions, phenomenological models are constructed by supplementing model theories to evaluate their theoretical findings against experimental data as well as to work them out for their experimentally ascertainable consequences. ${ }^{17}$ The essential differences between model theories of HEP and their various phenomenological models lie in the hypotheses, techniques and mechanisms used and the assumptions made with regard to the phenomena of interest. All these theoretical ingredients are more detailed and phenomena-specific in phenomenological models than in model theories. As a result, unlike model theories, phenomenological models are specific enough to offer predictions that are directly testable in particle physics experiments. And also, they are much more tractable for the analysis of experimental data as well as for the design of the experimental set up. On the other hand, theoretical elements accommodated by phenomenological models are applicable for a relatively narrow domain of phenomena, and thus serve the specific purpose of decoding model theories for their observable consequences only in this relatively narrow domain. Thus, one can say that phenomenological models have a relatively narrower range of applicability with respect to model theories of HEP.

To sum up, in this section, I have drawn a three-fold distinction in theorizing in HEP. The discussion suggests that in the context of HEP the term "theory" is used in a broad sense, so as to denote not just background theories and model theories but also phenomenological models. This is also compatible with the high-energy physicists' use of the term "theory". Another lesson this section suggests is that the above mentioned types of theories and models bring qualitatively distinct commitments in particle physics experiments; as they account for different aspects of sub-atomic phenomena in varying detail and scope. In the ensuing sections of the present paper, I shall discuss the philosophical implications of the above categorization of the theories of HEP for the notion of TLE. In the next section, I shall introduce the scattering-matrix research program (SMRP) which has been influential in HEP in the 1960s. Within the scope of this research program, I shall discuss the appropriateness of the above-mentioned categorization.

\footnotetext{
${ }^{16}$ See especially Hartmann 1999, where phenomenological model building in QCD is discussed. Hartman's case studies include the MIT Bag Model, the Nambu Jona-Lasinio Model, and the Chromodielectric Soliton Model.

${ }^{17}$ It is to be noted that this point gives support to Morrison's claim that the independence of phenomenological model building from high-level theories should be understood only as partial independence.
} 


\section{The Scattering-Matrix Approach to the Physics of Strong Interactions}

In the terminology of HEP, the term "total scattering cross-section" is essentially defined to be the measure of the scattering rate of the particles impinging on a target particle in terms of the scattering angle. One of the main tasks in HEP is to measure the total scattering cross section of the interactions between sub-atomic particles; as this provides a means of obtaining information about various features of sub-atomic phenomena. During the period following the World War II, the only theoretical tool used by particle physicists for the cross-section calculations was the Feynman diagram method invented by the American physicist Richard Feynman. ${ }^{18}$ This method employs what is called the perturbative approach of QFT that consists of a set of approximation techniques to calculate the scattering cross-section. Each term in the perturbation expansion of the scattering amplitude, which characterizes the probability of scattering in a given direction, corresponds to one of the intermediate states associated with the interaction under consideration. Each intermediate state is schematically represented by a diagram in which the straight lines and vertices signify the world lines of the particles and their mutual interactions, respectively. For instance, the Feynman diagram shown in Figure 1 represents a scattering process between the electron $\left(e^{-}\right)$and the positron $\left(e^{+}\right)$, where a virtual photon $(\gamma)$ is exchanged.

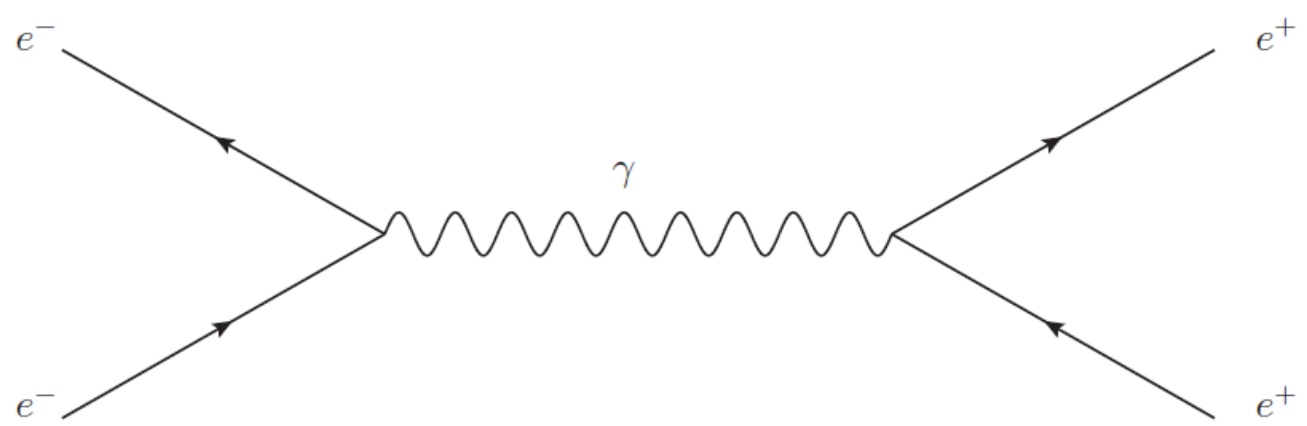

Figure 1: Feynman diagram of electron-positron scattering. (This diagram was drawn by using JaxoDraw; see Binosi and Theussl, 2004.)

In order to calculate the total scattering cross section for a certain nuclear process, one needs to sum up over the amplitudes of all the possible intermediate states. By the mid-1950s, Feynman diagram method has been very successful in the framework of QED. However, QFT's perturbative approach was not valid for performing cross section calculations associated with hadrons, which are distinguished by their strong interactions. In those cases, due to the fact that the coupling constant ${ }^{19}$ of the strong interaction is large, perturbation expansion yielded divergent terms, and no technique was available to extract observable quantities from the infinite results of the perturbation calculations; amounting to the commonly called "renormalization problem". ${ }^{20}$

\footnotetext{
${ }^{18}$ For a detailed history of Feynman diagrams, see Kaiser 2005.

${ }^{19}$ A coupling constant denotes the strength of an interaction.

${ }^{20}$ In QFT, the term "renormalization" refers to the collection of mathematical techniques used to extract finite, experimentally ascertainable quantities when the perturbation expansion yields divergent terms. Here, it is worth also mentioning that QED had also a renormalization problem, but it was solved before the 1950s; see, e.g., Pickering 1984, 65-68; and Schweber 1994, sec. 8.9.
} 
From the mid-1950s onwards, physicists' interest gradually shifted from QFT to the Scattering Matrix Theory (SMT), which had been proposed by Werner Heisenberg in his seminal 1943 papers (see Heisenberg 1943a, 1943b). ${ }^{21}$ In constructing SMT as an alternative theory of QFT, Heisenberg had a simple motivation that can be summarized as follows. In QFT, the basic entities are fields, rather than particles. Yet, fields are not directly detectable quantities. On the other hand, experiments in HEP are essentially scattering experiments where only particles can be detected. Heisenberg suggested that only those quantities that are experimentally ascertainable should enter the theories of HEP. Accordingly, he considered the scattering-matrix (Smatrix $)^{22}$ - the components of which are directly related to experimentally detectable quantities - as the fundamental dynamical quantity, rather than the Lagrangian. If one knows the S-matrix associated with a nuclear interaction, one can calculate almost all of its properties that can be tested by experimentation. However, in its form originally proposed by Heisenberg, SMT did not prove very useful for the study of strong interactions, as the S-matrix equations gave rise to an infinite set of coupled differential equations that were yet to be solved exactly.

The situation in HEP at the end of the 1950s was very desperate as far as the physics of strong interactions was concerned. Both QFT and SMT were beset by the technical difficulties that seemed insurmountable. ${ }^{23}$ In the early 1960s, a challenging proposal came from the Berkeley physicist Geoffrey Chew who was a strong adherent of SMT. Chew suggested that QFT was completely useless for the study of strong interactions and that it be abandoned and replaced by SMT. In contrast to the hierarchical taxonomic approach of QFT, Chew suggested that each strongly interacting particle should be treated equally. To this end, Chew and his collaborator Steven Frautschi proposed to supplement SMT by what they called the "bootstrap hypothesis" 24 that suggested a mutual generation mechanism for all particles interacting via the strong force - namely, the "bootstrap mechanism" (see Chew and Frautschi 1961a). According to this mechanism, no particle was considered "elementary"; rather each strongly interacting particle was conceived of as a composite (or bound state) of all the other strongly interacting particles. In Chew and his collaborators' words:

The strongly interacting particles are all dynamical structures that owe their existence to the same forces through which they mutually interact. In short, the strongly interacting particles are the creatures of the strong interaction. We refer to this as the "bootstrap" hypothesis. (Chew, Gell-Mann and Rosenfeld 1964, 93)

According to the bootstrap hypothesis, all strongly interacting particles are taken to be on the same footing in the sense that they bootstrap each other, which in turn enables them to generate themselves. Since the bootstrap mechanism demanded the equal treatment of all strongly interacting particles, Chew called this approach to the physics of strong interactions "nuclear democracy" (see Chew 1964, 105). ${ }^{25}$

\footnotetext{
${ }^{21}$ Also, for an early history—the years between 1942 and 1952—of SMT, see, e.g., Rechenberg 1989.

${ }^{22}$ In QM, the S-matrix is a mathematical quantity whose elements are called "scattering amplitudes" that denote the probability distribution of the transitions between the initial and the final states of a scattering process. Note also that the S-matrix was first introduced by John Wheeler as a calculational tool for nuclear physics (Wheeler 1937).

${ }^{23}$ Since my focus in this paper is experimentation in the 1960s in the physics of strong interactions, I here leave out the discussion of the developments in SMT in the 1950s; for this see Cushing 1990; and Cao 2010.

${ }^{24}$ See also Chew 1961, 1962, 1963.

${ }^{25}$ Also note that Chew's bootstrap hypothesis and his notion of nuclear democracy have been extensively studied in the literature of history and philosophy of modern physics; see, e.g., Gale 1974; Freundlich 1980; Cushing 1985;
} 
SMT as developed by Chew and his collaborators was basically a theory of strong interactions. This new form of SMT, referred to as the analytic SMT, was free of some of the technical difficulties besetting its original formulation by Heisenberg. ${ }^{26}$ Yet, the analytic SMT was still far from offering experimentally testable predictions. Nor was it amenable to the analysis of experimental data. The next task in the SMRP was to apply the analytic SMT to experiments. To this end, Chew and his collaborators Steven Frautschi and Stanley Mandelstam considered a mathematical technique previously proposed by the Italian theoretical physicist Tullio Regge (see Regge 1959, 1960). The basic idea of the Regge technique is that the scattering amplitude in a scattering process is a complex-valued analytic function of the angular momentum. ${ }^{27}$ Accordingly, the Regge technique suggests that scattering calculations should be performed in terms of energy and momentum variables on the complex plane ${ }^{28}$, instead of momentum transfer ${ }^{29}$ values. ${ }^{30}$ By using this technique, Regge had established in the nonrelativistic regime a bound on the asymptotic behaviors of the elastic scattering amplitudes for large momentum transfer values. ${ }^{31}$ The Regge technique was imported by Chew and his collaborators into the relativistic regime and incorporated into the bootstrap mechanism as a boundary condition at small momentum transfer values corresponding to small scattering angles (see Chew and Frautschi 1961b; and Chew, Frautschi and Mandelstam 1962).

This further elaboration rescued the analytic SMT from some of the technical difficulties it previously faced, especially from the ones encountered in solving the bootstrap equations. More importantly, for the future of the SMRP, this new form of the analytic SMT - typically referred to as the "Regge theory or model" in the physics literature (see, e.g., Collins 1977-was able to yield experimentally testable predictions about hadron-hadron scatterings. The key prediction of the Regge theory was that at high energies and small scattering angles the hadron-hadron scattering cross section would fall rapidly with the increase of the momentum transfer during scattering (see Chew and Frautschi 1961a; Frautschi, Gell-Mann and Zachariasen 1962). This meant that the scattering cross section would be low at large scattering angles. By the mid 1960s, the Regge theory proved very useful in the experimental studies of hadronic interactions. As shall be seen in sec. 6.1, the match between the predictions of the Regge theory and the results of the various experiments played an important role in its wide acceptance within the HEP community.

Before I close this section, I shall look at the SMRP through the categorization of the theories of HEP that I brought up in the previous section. The following might be helpful to see

Cao 1991; Kaiser 2002; Redhead 2005. Chew himself also wrote several reflective articles; see, e.g., Chew 1968, $1970,1989$.

${ }^{26}$ This was ensured by the conditions of "maximal analyticity", "unitarity" and "crossing". For the discussion of these concepts, see Cao 2010, sec. 2. It is also worth noting that the fact that the analytic SMT satisfied the foregoing conditions was seen as its success against QFT in accounting for strong interactions and thereby played as a driving force in its initial acceptance.

${ }^{27}$ An analytic function is one that is differentiable at all orders at each point in its domain. A complex function is one that is of the form $z=x+i y$, where $x$ and $y$ are real numbers, and $i$ is called the "imaginary number" whose square is, by definition, equal to -1 .

${ }^{28}$ The complex plane (also called z-plane) is a geometrical representation of complex numbers, where the $x$ and $y$ axes respectively represent the real and imaginary parts of complex numbers.

${ }^{29}$ This is the amount of momentum transferred from one particle to another during a collision process.

${ }^{30}$ See Pickering 1984, 75.

${ }^{31}$ In an elastic scattering (collision) interacting particles bounce off one another with very little momentum exchange between them, so that the total kinetic energy of the particle is conversed. By contrast, in an inelastic scattering the momentum transfer is large and the total kinetic energy of the particle is not conserved. 
how the original SMT and the analytic SMT stand to each other. SMT, as originally proposed by Heisenberg, is a quantum theory of scattering phenomena. Its theoretical framework involves rules and principles that enable more elaborate theories of HEP to be constructed. The analytic SMT was constructed in this framework as a theory of strong interactions. In this sense, the original SMT can be said to play the role of a background theory in the SMRP — as does QFT in the context of gauge theories of particle physics. However, unlike the original SMT, the analytic SMT is able to account for how sub-atomic particles interact via the strong force. It does this by invoking the principle of nuclear democracy as a guiding principle, and more importantly by appealing to the bootstrap mechanism that is exclusively applicable to its target phenomena, i.e., strong interactions. Therefore, I identify the original SMT as the background theory of the SMRP and the analytic SMT as the model theory of strong interactions in this research program.

As we have seen above, the analytic SMT by itself does not offer experimentally testable predictions about its target phenomena, i.e., strong interactions. Consequently, in the SMRP, theory enters experimentation largely through the Regge theory. As I have mentioned earlier, the successful predictions of the Regge theory concerning hadronic processes expedited the establishment of the SMRP. The Regge theory is an elaboration of the analytic SMT in the sense that it was constructed by supplementing the latter with the Regge technique. Much of the predictive power of the Regge theory is due to this technique, which governs the asymptotic behavior of the elastic scattering amplitude at high energies. However, this advantage comes with a price. The Regge theory has a relatively narrow range of applicability as compared to the analytic SMT; for the Regge technique works only for elastic types of scattering phenomena. Recall that in the previous section in drawing the distinction between model theories of HEP and their phenomenological models, I have maintained the view that the latter are constructed primarily for the purpose of extracting testable predictions from model theories which, when taken all by themselves, do not typically offer testable predictions about their target phenomena. Also, I have argued, as compared to their corresponding model theories, phenomenological models have narrower range of applicability; as their structural elements-which come in the form of mechanisms, hypotheses and techniques - apply only to the sub-domains of the target phenomena of their model theories. Summing up, it is clear that both the way the Regge theory was constructed out of the analytic SMT and the role it played in the SMRP perfectly illustrate the distinction I have previously drawn between model theories of HEP and their phenomenological models. This in turn suggests the Regge theory as a phenomenological model of the analytic SMT in the SMRP.

The success of the Regge theory in the description of hadron-hadron scattering processes hindered the study of backward scattering region-corresponding to large momentum transfer regime - for other types of interactions, such as electron-proton scatterings. Until the mid 1960s, the analytic S-matrix theory and its phenomenological application, namely the Regge theory, gradually became accepted as the dominant view within the HEP community. ${ }^{32}$ The first challenge against this dominant view came from the quark model, which I shall consider in the next section.

\footnotetext{
${ }^{32}$ See Pickering 1984, sec. 3.4.
} 


\section{The Quark Model and the Current Algebra Approach to the Physics of Strong Interactions}

Using the conceptual tools of QFT, the American physicist Murray Gell-Mann proposed an alternative approach to the physics of strong interactions that is now referred to as "current algebra" in the literature of HEP (Gell-Mann 1962). Strongly interacting particles, namely hadrons, also undergo electromagnetic and weak interactions. The current algebra approach is concerned with those interactions of strongly interacting particles, and it is based on symmetry considerations, which had been previously applied to the domain of weak and electromagnetic interactions. ${ }^{33}$ Gell-Mann suggested that, like electromagnetic and weak interactions, strong interactions could be modeled on the basis of a symmetry principle. He thereby proposed that strong interactions could be described by using the SU(3) algebra (Gell-Mann 1962). ${ }^{34}$

In a two-page article, Gell-Mann proposed a model of hadrons that illustrated the essential features of the current algebra approach, which he had previously developed for strong interactions (Gell-Mann 1964). ${ }^{35}$ In the same year, the Russian-born American physicist George Zweig independently proposed a similar model (Zweig 1964a, 1964b). According to those models, hadrons were composed of more fundamental particles, which Gell-Mann called "quarks" and Zweig "aces". ${ }^{36}$ Both physicists suggested that the constituent particles were of three distinct types, namely, what are today referred to as "up", "down" and "strange" quarks; each carrying a spin of $1 / 2$ and fractional charge of respectively $2 e / 3,-e / 3$ and $-e / 3$, where $e$ denotes the electric charge of the electron.$^{37}$ It is worth noting that the proposal of fractional charges was in great opposition with the general scientific belief, the root of which dated back to Robert Millikan's oil drop experiments ${ }^{38}$, which had demonstrated that electric charge existed in nature as integral multiples of the charge of the electron. ${ }^{39}$

The quark model posed a real challenge to the HEP community that was highly satisfied by the success of the Regge theory in the description of strong interactions. The quark proposal was bringing back again the idea that some particles were more "fundamental" than the others. Obviously, this was an idea to which the proponents of SMT, who were subscribed to the view of "nuclear democracy", were highly opposed. Yet, the quark model was successful in solving some symmetry problems that the analytic SMT could not account for. ${ }^{40}$

The first real reaction of the HEP community towards the quark model was to look for particles of fractional charge in accelerator experiments. The second alternative was to look for quarks in cosmic rays. And, the third option was to perform more elaborate versions of Millikan's oil drop experiments in order to search for fractional electric charges. However, none of these options produced any evidence for the existence of quarks. ${ }^{41}$ The lack of empirical

\footnotetext{
${ }^{33}$ See, e.g., Cao 1997, chap. 8; Pickering 1984, chap. 6.

${ }^{34}$ For details, see Pickering 1984, sec. 4.3; and Cao 2010, chap. 2.

${ }^{35}$ For details concerning the relation between Gell-Mann's quark model and the algebra he developed, see especially Cao 2010, sec. 2.3.

${ }^{36}$ At the present time, in HEP, Gell-Mann's nomenclature is used and the hadronic constituents are referred to as "quarks".

${ }^{37}$ For further differences and similarities between the types of quarks, see, e.g., Griffiths 2008, chap. 1.

${ }^{38}$ For details, see Franklin 1997.

${ }^{39}$ For more details on Gell-Mann's and Zweig's proposals, as well as on their differences, see Pickering 1984, chap. 4.

${ }^{40}$ For example, the quark model explained why triplet, sextet, and 27-plets of SU(3) symmetry were absent.

${ }^{41}$ For a review of quark search experiments in the 1960s, see, e.g., Jones 1977.
} 
evidence was an important reason behind the reluctance of the HEP community towards the acceptance of the quark model. Apart from the lack of empirical evidence, it is noteworthy that, as pointed out by both Kendall ${ }^{42}$ and Bjorken ${ }^{43}$, at the time the quark proposal was put forward the concept of "integer charge" was very prevalent among the HEP community; greatly precluding the acceptance of quarks as real particles. ${ }^{44}$

An historical extension of the current algebra approach was the construction of "sum rules" 45 . On the basis of Gell-Mann's current algebra, the American physicist Stephen Adler derived a sum rule for the inelastic neutrino ${ }^{46}$ scattering from nucleon (Adler 1966). By drawing on the sum rule derived by Adler and the current algebra technique, the SLAC theorist James Bjorken derived a lower bound for backward inelastic electron-nucleon scattering that suggested a large amount of cross section at high momentum transfer. ${ }^{47}$ Based upon this result and current algebra, Bjorken concluded that "the total backward scattering at fixed large [momentum transfer] [was] predicted to be at least as great as that from a point Dirac particle with charge \pm e/2" (Bjorken 1967b, 1767). At the Varenna International School of Physics (Varenna, July $17-$ $29,1967)$, Bjorken interpreted the same result as suggesting that backward inelastic electronnucleon "scattering is the sum of the scattering from the Dirac moments of the point spin- $1 / 2$ constituents (if they exist)". 48

\section{A Closer Look at the Theory-Experiment Relationships in the Physics of Strong Interactions in the 1960s}

I have previously suggested a categorization of the theories of HEP. But, I have not yet discussed the philosophical implications of this categorization for scientific experimentation. To this end, in this section, I shall examine experiments from the history of HEP in terms of theoryexperiment relationship. My discussion is also intended to highlight some major theoretical developments that went together with experimentation. In contrast to an undifferentiated theoryexperiment relationship suggested by the theory-dominated view of scientific experimentation, I shall seek to argue that the aforementioned categorization of the theories of HEP indicate that "theory" and experiment may interact in different ways and that this in turn brings about the existence of different senses of TLE in the practice of HEP.

\section{1 The Elastic Proton-Proton Scattering Experiments}

As mentioned earlier, the Regge theory offers experimentally testable predictions about strong interactions. According to this theory, the hadron-hadron differential scattering cross section

\footnotetext{
${ }^{42}$ See Kendall 1991, 599.

${ }^{43}$ Bjorken (personal communication, March 20, 2012).

${ }^{44}$ In a similar vein, Friedman $(2012,470)$ notes: "The general point of view in 1966 was that quarks were most likely just mathematical representations: useful but not real. The real picture of particles was that they have diffuse substructures and no elementary building blocks".

${ }^{45}$ In QM, a sum rule gives the total transition rate from one state to another in a scattering process.

${ }^{46}$ Neutrinos are elementary particles that carry no charge but spin-1/2 and that undergo weak interactions.

${ }^{47}$ See equations (6.28) and (5) in Bjorken 1967d ,1967b, respectively. See also equations (1) and (6.21) in Bjorken 1966a, 1966b. For more historical and physical details, see Cao 2010, sec. 3.3.

${ }^{48}$ It is interesting to note that, like Gell-Mann and Zweig, Bjorken here cautiously speaks of the possibility of the existence of non-integer spin-1/2 constituents of the nucleon.
} 
$(1 / k)^{2} d \sigma / d \Omega$ - whose integration over the entire solid angle $\Omega$ yields the total scattering cross section $\sigma$-obeys the following mathematical expression:

$$
\frac{1}{k^{2}} \frac{d \sigma}{d \Omega} \approx F(t)\left(\frac{S}{2 M^{2}}\right)^{2 L(t)-2},
$$

where $t=-2 k^{2}(1-\cos \theta)$ is the negative of the square of the four-momentum transfer and $\theta$ is the scattering angle; $S$ denotes the square of the center of mass energy; $L(t)$ represents the change in the angular momentum; and $M$ stands for the hadron mass. $F(t)$ denotes the scattering amplitude, and at high energies, it is an exponentially decreasing function of $t$, which is always negative in the physical region, and $L(t)$ is taken to be less than 1 . The Regge theory makes the following predictions for elastic hadron-hadron scattering cross section: (1) the scattering cross section should decrease exponentially with increasing momentum transfer in a scattering process; and (2) the width of the forward scattering peak should shrink logarithmically with increasing scattering energy. ${ }^{49}$ These two predictions of the Regge theory concern two different aspects of the hadron-hadron scattering cross section. The first prediction indicates that the rate of the scattered particles would diminish as the momentum transfer between the scattered particles and the target increases. The second prediction indicates that the rate of the scattered particles would also diminish as the energy at which the particles scatter from the target increases. Soon after the formulation of the Regge theory, a number of elastic proton-proton scattering experiments ${ }^{50}$ were launched with the aim to test the above predictions of the Regge theory. This was also clearly pointed out in the publications of the experimental groups that performed those experiments, as the below passage exemplifies:

This experiment is part of a program to study basic strong interactions in the energy range $\ldots \sim 10-20 \mathrm{BeV}$. These measurements are of great interest due to the striking predictions made by Regge pole theory and allow a critical evaluation of the theory. If the energy is sufficiently high ... one would expect — as predicted by Chew and Frautschi and others - a shrinkage of [the scattering cross section] corresponding at low [momentum transfer]. (Foley et al. 1962, 376)

At this point, I shall note that the fact that the proton-proton scattering experiments were planned and launched by taking into account the predictions of the Regge theory is not enough to conclude that these experiments are theory-laden in the sense of TDE. It is to be recalled that TDE, as I have defined in the preceding discussion, requires also other stages of the experimental process - such as experimental design, data acquisition, data analysis as well as data interpretation - to be carried out in a theory-regulated way. Now, let us see how the stages of experimental design and data acquisition were carried out in proton-proton scattering experiments. First, it is to be noted that the fact that these experiments were aimed to test the predictions of the Regge theory had a significant impact on the selection of the phenomena to be scrutinized. Only the forward scattering region, which corresponds to elastic scatterings, was probed in the proton-proton scattering experiments; so that data were only taken in this region. It is also to be noted that the forward scattering region was the only region that was relevant to the

\footnotetext{
${ }^{49}$ For technical details, see Cushing 1990, sec. 6.5.

${ }^{50}$ See, e.g., Diddens et al. 1962; Foley et al. 1962a; Fujii et al. 1962.
} 
predictions of the Regge theory. As a result, the backward scattering region that corresponds to inelastic scatterings of protons was disregarded. This points out that the instrumental arrangement and the data-acquisition process in the proton-proton scattering experiments were specific to the phenomena relevant to the testing of the Regge theory. Therefore, I conclude, both the experimental design and the data-taking procedures in the proton-proton scattering experiments were arranged specifically to test the aforementioned predictions of the Regge theory.

The impact of the Regge theory on the proton-proton scattering experiments is also manifest in the way the collected data were analyzed. The data-analysis was aimed to extract the information that would enable to draw conclusions about the predictions of the Regge theory. More specifically, as indicated in Figure 2, the collected cross section data were plotted against the momentum transfer at different energy values. It was found that the width of the forward scattering peak decreased with increasing energy, and that its tail fell off exponentially with increasing momentum transfer. That is, the results of the elastic proton-proton scattering experiments were found to be compatible with the predictions of the Regge theory.

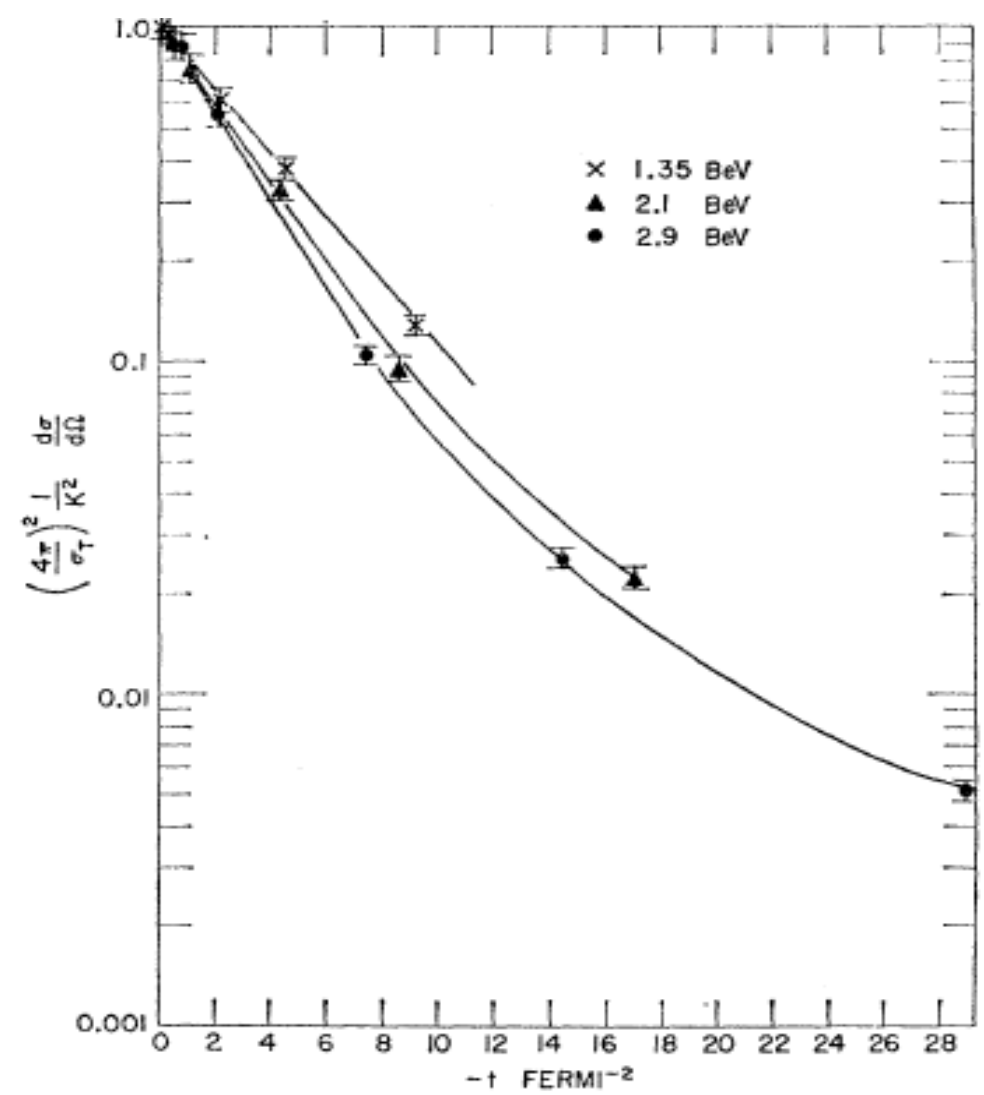

Figure 2: The scattering cross section at various energies as a function of momentum transfer. From Fujii et al. 1962.

Moreover, the collected data were also used to determine the numerical values of the function $F(t)$ at different momentum transfer values. Note that $F(t)$ is a mathematical function that represents the high energy behavior of the scattering cross section. In this sense, its numerical determination requires the mathematical formalism of the Regge theory that connects it to the cross section, energy and momentum values, which are directly measurable physical quantities in a scattering experiment. The numerical results of the function $F(t)$ were found to exhibit an 
exponentially decaying behavior with increasing momentum transfer $t$, while remaining virtually independent of energy values. This result was also compatible with the theoretical framework of the Regge theory, in which $F(t)$ was defined to be only a function of the momentum transfer $t$. The elastic proton-proton scattering experiments ended with the conclusion that the elastic proton-proton scattering cross section exhibited a behavior as predicted by the Regge theory. My conclusion regarding the data-analysis in the proton-proton scattering experiments is that it was more narrowly intended as not aiming to the detection of unforeseen effects that might be hidden in the elastic scattering data, but solely to the testing of the predictions of the Regge theory.

The above discussion indicates that the successive stages of the elastic proton-proton scattering experiments were carried out by appealing to the theoretical framework of the Regge theory. In other words, the Regge theory can be said to have provided a definite road map for the elastic proton-proton scattering experiments to proceed from their initial design up to their final stage. This conclusion allows me to call these experiments theory-laden in the strong sense of TLE, i.e., TDE.

\subsection{The Deep-Inelastic Electron-Proton Scattering Experiments}

In this subsection, I shall examine the deep-inelastic electron-proton scattering (DIS) experiments that were performed at the Stanford Linear Accelerator Center (SLAC) between the years of 1967 and 1973. ${ }^{51}$ At the outset, I shall give a brief overview of the earlier developments in experimental HEP. The elastic scattering of electrons from nucleons (protons or neutrons) was first studied in the 1950s by a group of physicists led by Robert Hofstadter from Stanford University. The data obtained at the end of a series of experiments ${ }^{52}$ showed that the elastic scattering cross section decreased sharply with increasing momentum transfer. This strengthened the belief that the proton had an extended internal structure. The data also revealed that the size of the nucleons was roughly $10^{-1} \mathrm{~cm}$. These experimental findings marked the beginning of a new era for the search of the inner structure of the proton, while bringing Hofstadter the Nobel Prize in 1961. It is to be noted that the electron beam energies at that time were still low to be able to study the inelastic scattering of electrons from nucleons. ${ }^{53}$ This was the future research direction in experimental HEP towards the mid-1960s. But, the HEP community was urgently in need of a better accelerator technology that would allow the study of the inelastic scattering of electrons from nucleons.

In 1961, the U.S. Congress approved the proposal of Stanford University to construct a linear accelerator that could accelerate electrons up to $22 \mathrm{GeV}$. The construction of the linear accelerator at the SLAC was completed in $1966 .{ }^{54}$ Soon after, in early 1967 , the collaboration consisting of physicists from SLAC, Massachusetts Institute of Technology (MIT) and California Institute of Technology (Caltech) embarked on a project primarily aiming at the analysis of the inelastic electron-proton scattering. Jerome Friedman (MIT) ${ }^{55}$ was one of the co-leaders of this

\footnotetext{
${ }^{51}$ Since I am mainly concerned with the epistemological aspects of the DIS experiments, my discussion is not intended to give a detailed history of these experiments. A detailed narrative of the DIS experiments was provided by Michael Riordan (Riordan 1987, chaps. 6-8), who was also involved in these experiments as a physicist.

${ }^{52}$ Hofstadter and McAllister 1955; Hofstadter 1956.

${ }^{53}$ See, Pickering 1984, sec. 2.3 for a review of the history of particle accelerators.

${ }^{54}$ For the history of the construction of the SLAC linear accelerator, see Neal 1967.

55 Jerome Friedman (MIT), Henry Kendall (MIT) and Richard Taylor (SLAC) were the co-leaders of the project and they were jointly awarded the Nobel Prize in physics in 1990 for the DIS experiments.
} 
project and summarized the intended scope of this project as follows in his Nobel Lecture of December 8, 1990:

The main purpose of the inelastic program was to study the electroproduction of resonances $\left[{ }^{56}\right]$ as a function of momentum transfer. It was thought that higher-mass resonances might become more prominent when excited with virtual photons, and it was our intent to search for these at the very highest masses that could be reached. (Friedman 1991, 615)

As a first step, the Caltech-MIT-SLAC collaboration studied the elastic scattering of electrons from the proton target. The data obtained showed that the scattering cross section fell off rapidly as the momentum transfer during the scattering increased. ${ }^{57}$ This result was compatible with the expectation of the collaboration. According to the project-proposal, the next step was to study the inelastic scattering of electrons from the proton target. At this stage of the project, Caltech group dropped out of the collaboration, thinking that the planned inelastic experiments would merely duplicate the known outcomes of the previous elastic electron-proton scattering experiments. ${ }^{58}$

As a result, the inelastic part of the project was undertaken by the MIT-SLAC collaboration. "For completeness [the collaboration] also wanted to look at the inelastic continuum, since this was a new energy region which had not been previously explored" (Friedman 1991, 615). ${ }^{59}$ For the collaboration, even though the inelastic continuum, i.e., deepinelastic scattering region ${ }^{60}$, was a novel scattering region to explore, their expectation was that the DIS experiments would not yield results that would challenge the widely held belief that "the nucleon was the extended object found in elastic electron scattering but with the diffuse internal structure seen in ... proton scattering." ${ }^{\circ 1}$ As pointed out by Friedman $(2012,470)$ "the general point of view was that hadrons did not have elementary constituents, namely point-like constituents that were described by a field theory. A consequence of this picture was that hadrons would have diffuse substructures and no elementary building blocks". However, this, more or less, standard view of the nucleon was not suggested to the experimentalist group by a wellestablished theoretical account. However, the group had good reasons for their commitment to that view of nucleon structure. First, it was suggested by the previous elastic electron-proton scattering experiments. ${ }^{62}$ On the theoretical side, the quark proposal was the only challenge; but,

\footnotetext{
${ }^{56}$ In HEP, "resonances" are defined to be intermediate states associated with the products of the scattering event and are signaled by the presence of "peaks" in the cross section data. In the inelastic scattering project at SLAC, resonances were produced by the emission of electrons into the proton target; a process called "electroproduction". ${ }^{57}$ See Coward et al., 1968.

${ }^{58}$ This was expressed by the Caltech experimenter Barry Barish in different interviews; see Pickering Pickering 1984, 152, note: 7; and the interview with Shirley Cohen at the URL: http://oralhistories.library.caltech.edu/178/01/Barish_OHO.pdf.

${ }^{59}$ Elsewhere, Friedman also stated: "The program committee was not very happy about our measuring the inelastic continuum. They thought it was really a waste of time ... In fact, the continuum had never really been studied seriously even at lower energies. We weren't sure what we were looking for; but we decided that since nobody in the past had ever looked at the continuum and this was a new energy range, we should do it" (Friedman 2012, 472). ${ }^{60}$ Note that the "inelastic continuum", also referred to as "deep inelastic scattering region" or "backward scattering region", is the region in a scattering experiment where deep inelastic scattering processes occur at wide scattering angles (corresponding to large momentum transfer values) at sufficiently high energies. In these processes, particles can penetrate deeply into the target particle, resulting in the formation of debris of the target particle.

${ }^{61}$ Kendall 1991.

${ }^{62}$ Hofstadter and McAllister 1955.
} 
as has been mentioned previously, the quark model had not received any support from experiments. The foregoing commitment led the experimentalist group to the supposition that the proton's inner structure could not serve to scatter the impinging electrons at large angles. This was also pointed out by Friedman in his Nobel Lecture as follows:

When the experiment was planned, there was no clear theoretical picture of what to expect. The observations of Hofstadter (McAllister and Hofstadter, 1956) in his pioneering studies of elastic electron scattering from the proton showed that the proton had a size of $10^{-1} \mathrm{~cm}$ and a smooth charge distribution. This result, plus the theoretical framework that was most widely accepted at the same time, suggested to our group when the experiment was planned that the deep-inelastic electron-proton cross sections would fall rapidly with increasing [momentum transfer during the scattering.] (Friedman 1991, 616)

When the inelastic program began in September of 1967, the theoretical landscape in the physics of strong interactions was quite unsettled. The Regge theory was considered to be the mainstream approach to the study of hadron-hadron scattering processes. This was largely due to its predictions that were confirmed in the elastic hadron-hadron scattering experiments. But, an important shortcoming of the Regge theory was that it was not able to account for inelastic scattering processes between hadrons. ${ }^{63}$ Furthermore, QFT's perturbative approach to strong interactions was beset by the problem of renormalization, which hindered the study of hadronic processes. The only theoretical result concerning backward electron-proton inelastic scattering was the lower bound obtained by Bjorken on the basis of current algebra just before the inelastic part of the SLAC project began. As we have previously seen, this result was suggestive of a pointlike structure of the nucleon. At this point, it is worth pointing out that at the time the SLAC project was underway, Bjorken was working in the theory-division of the SLAC. As the following passage from Friedman's Nobel Lecture indicates, the members of the MIT-SLAC collaboration apparently heard about Bjorken's work on electron-proton scattering prior to the inelastic part of the project; however, they did not take Bjorken's result into account when they began the DIS experiments:

Bjorken's result made little impression on us at the time. Perhaps it was because these results were based on current algebra, which we found highly esoteric, or perhaps it was that we were very much steeped in the physics of the time, which suggested that hadrons were extended objects with diffuse substructures. (Friedman 1991, 618)

Friedman's words suggest that two factors directly contributed to the dismissal of Bjorken's proposal by the MIT-SLAC collaboration. First, the members of the collaboration did not understand current algebra well enough to be able to judge and recognize the significance and the implications of Bjorken's proposal. Second, Bjorken's proposal was in opposition with the common thinking among the HEP community about the inner structure of hadrons to which the group members were strongly committed; thereby disinclining them to consider Bjorken's proposal. Thus, it would be correct to say, as Kendall also remarked in his Nobel Prize Lecture

\footnotetext{
${ }^{63}$ Note that the electron-proton scattering is not a hadron-hadron type scattering, as electrons are not hadrons. Electrons are part of another class of particles called "leptons". For the basic features of leptons, see, e.g., Griffiths 2008, 74.
} 
of December 8, 1990, "there was no consideration that possible point-like substructure of the nucleon might be observable in electron scattering during the planning and design of the electron scattering facility" (Kendall 1991, 600). In a private communication, Friedman expressed a similar view:

Our initial experiment was planned and carried out without regard to any prediction from the world of theory. It was meant to be an exploratory investigation of electro-production of nucleon resonances and of highly inelastic scattering from the nucleon in a new energy range. We had hoped that such a study might shed some light on the structure of the nucleon. It was really a fishing expedition. ${ }^{64}$

The DIS experiments were carried out by the MIT-SLAC collaboration led by Richard Taylor (from MIT) and Jerome Friedman and Henry Kendall (from SLAC). In contrast to the expectation of the group, in the deep-inelastic scattering region corresponding to large scattering angles and high energies, the scattering cross sections were found not to change considerably and remained close to those at small angles. In other words, the inelastic scattering data revealed that the dependence of inelastic scattering cross section on momentum transfer was rather weak. ${ }^{65}$ Figure 3 depicts this feature of electron-proton inelastic scattering spectrum obtained at the DIS experiments.

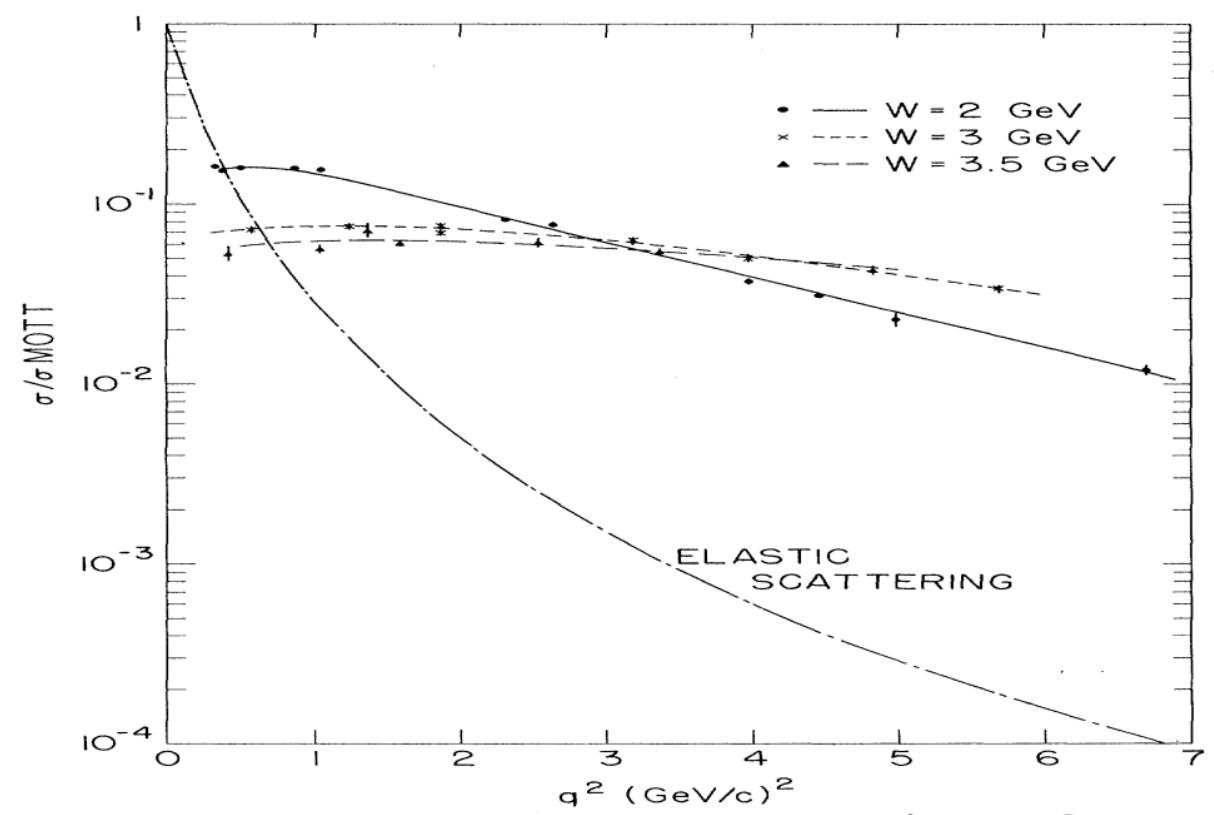

Figure 3: SLAC inelastic cross sections $(\sigma)$ as a function of momentum-transfer $\left(q^{2}\right)$. The upper curves represent inelastic cross-section measurements, and the lowest one represents the data from elastic scattering. From Breidenbach et al. 1969.

The historical context in which the DIS experiments were launched indicates that the MIT-SLAC collaboration did not take into account Bjorken's 1967 result (concerning the backward electronproton inelastic scattering) that was the only theoretical result relevant to the aims of the DIS

${ }^{64}$ Friedman (personal communication, October 4, 2007).

${ }^{65}$ Bloom et al. 1969. 
experiments. Therefore, it would be correct to conclude that the stages of planning, design and data acquisition of the DIS experiments were carried out without recourse to the guidance of any workable theory or phenomenological model with regard to the scattering processes under scrutiny. Rather, those stages were geared towards the exploration of the forward scattering region as well as the backward region, which had not been probed in previous scattering experiments. Here, what I mean by "exploration" can be understood as systematically looking for patterns of regularities in the scattering cross section spectrum and extracting from them the information as to the dependency of inelastic scattering cross section on momentum transfer. It is to be noted that the fact that the experimentalist group did not have recourse to the guidance of any theoretical account as to what the scattering of electrons from the proton target would yield suggests that the DIS experiments were not driven by any theory or model in the sense of TDE. This is also manifest in the design of these experiments. Unlike the case of the earlier elastic proton-proton scattering experiments examined in the previous sub-section, the experimental design of the DIS experiments was not specific to any theory. Even though the experimentalist group had a clear expectation about the outcome of these experiments, namely that scattering cross section would be low in backward scattering region, their expectation was not decisive in the instrumental arrangement. It is to be noted that the instrumental arrangement in the DIS experiments was not intended to solely serve the expectation of the experimentalist group; but rather it enabled to probe the backward scattering region which was previously considered by the majority of the HEP community to be an uninteresting region for scattering experiments.

In order to understand how the data analysis was carried out in the DIS experiments, let us have a closer look at how the collected cross section data were processed in these experiments. The first unexpected feature of cross section data was discovered when the data were plotted as a function of the square of the four-momentum transfer, i.e., $\mathrm{q}^{2}=2 \mathrm{EE}^{\prime}(1-\cos \theta)$ for constant values of the invariant mass of the recoiling target system defined as $W=\sqrt{2 M\left(E-E^{\prime}\right)+M^{2}-q^{2}}$, where $E$ is the energy of the incident electron, $E^{\prime}$ is the energy of the scattered electron, $\theta$ is the scattering angle and $M$ is the mass of the proton. It was found that as $W$ increases, the $q^{2}$ dependence of cross section appeared to decrease, as indicated in Figure 3. It is to be noted that no theoretical prediction of what the scattering cross section data would look like as a function of $q^{2}$ and $W$ was considered prior to the dataacquisition stage of the DIS experiments. In this sense, the above finding of the MIT-SLAC collaboration should be regarded as an experimental discovery rather than an experimental test of a previously stated theoretical result. This suggests that, unlike the case of the earlier elastic proton-proton scattering experiments, the data analysis in the DIS experiments was not intended to the verification or disproval of any theory whatsoever. Nor was it directed towards discovering further empirical consequences of an existing theory. This discussion suggests that, like the earlier stages, the data analysis stage concerning the weak momentum transfer dependence of cross section data was carried out without having recourse to the guidance of any workable theory or phenomenological model.

Another unexpected and exciting feature of the inelastic electron-proton scattering data was understood by following a suggestion made by Bjorken. In 1964, Sidney Drell and John Walecka from Stanford University provided a formulation of the inelastic electron-proton scattering cross section by using the concept of "structure function." ${ }^{66}$ In this formulation, the total cross section

\footnotetext{
${ }^{66}$ This is also called "form factor". It describes the distribution of charge and magnetization within nucleons. For an extensive discussion on form factors, see Falkenburg 2007, chap. 4.
} 
is taken to be a function of the structure functions $W 1$ and $W 2$ that are in turn functions of the variables $q^{2}$ and $v$ representing the momentum transfer squared and energy lost of the electron during the scattering, respectively (Drell and Walecka 1964). Bjorken elaborated on this formalism and suggested what is now referred to as the "Bjorken scaling" of the electron-proton deep-inelastic scattering; namely, in the deep-inelastic scattering regime where both $q^{2}$ and $v$ become sufficiently large, $W_{1}$ and the product $v W_{2}$ depend only on the ratio $\omega=M v / q^{2}$ (where $M$ stands for the proton mass, and $\omega$ is the dimensionless scaling variable), rather than $q^{2}$ and $v$ independently. ${ }^{67}$ During the analysis of the inelastic scattering cross section data, Bjorken suggested, through dialogue with Kendall ${ }^{68}$, to the experimentalist group to analyze the scattering cross section data to check the scaling hypothesis. ${ }^{69}$ Upon this suggestion, the experimentalist group plotted $W_{1}$ and $v W_{2}$ as a function of $\omega$ and determined that the scattering cross section data, to a good approximation, exhibited scaling behavior in the way suggested by Bjorken. ${ }^{70}$ It is to be noted here that since the scaling variable $\omega$ is dimensionless the scaling behavior of the deep-inelastic electron-proton scattering data just described indicates that there is no absolute length or energy scale characterizing the scattering process; thereby suggesting that, in the deep-inelastic scattering regime, electrons can scatter off the proton target also at large scattering angles corresponding to higher momentum transfer values.

I shall close this sub-section with a brief discussion of the role of theoretical considerations in the DIS experiments. At this point, the following quotation from Friedman is worth mentioning:

The comparison [of the cross section data] with scaling was really an afterthought. None of us understood what the physical significance of scaling was at that time. ${ }^{71}$

Friedman's words suggest that Bjorken's explanation of scaling, like his inequality for the inelastic electron-proton scattering cross section, was not taken into account by the MIT-SLAC collaboration until the cross section data were acquired and analyzed and its weak momentum transfer dependence was observed. Therefore, one can conclude that in the DIS experiments Bjorken's account was appealed to only in the later part of the data analysis and interpretation stage that revealed the scaling feature of the experimental data. ${ }^{72}$ It is to be remembered that TDE, as I have previously defined, requires all successive stages of experimentation to be guided by the theoretical accounts of the target phenomena. Therefore, the fact that the scaling feature of the cross section data was discovered following Bjorken's suggestion does not render the DIS experiments theory-laden in the sense of TDE. It is also to be noted that a variety of other theoretical considerations were also relied upon to carry out these experiments. First, since these

\footnotetext{
${ }^{67}$ For the scaling hypothesis, see Bjorken 1969. It is worth pointing out that, as noted in Bjorken 2003, 1-4, the scaling hypothesis was first formulated by Bjorken in an incomplete, unpublished manuscript prior to the DIS experiments; see Bjorken 1967a.

${ }^{68}$ Friedman (personal communication, March 28, 2012).

${ }^{69}$ See Friedman 1991, footnote 3; Kendall 1991, 609; Taylor 2001, 233.

${ }^{70}$ Breidenbach et al. 1969; and Miller et al. 1972. Note that the analysis in the first paper was restricted to relatively smaller values of $q^{2}$ and $v$. The scaling feature of the cross section data was later verified for larger values by Miller et al. 1972.

${ }^{71}$ Friedman (personal communication, October 4, 2007).

${ }^{72}$ It is worth noting that this also illustrates Radder's claim that the later or immediate significance of experiments is affected by the theoretical context.
} 
are scattering experiments, detectors were used to detect and determine the number of scattered electrons per scattering angle. In this way, the ratio of the flux of the scattered electrons to the flux of the incident electrons was calculated. Experimenters identified this ratio as the total scattering cross section of the interaction between incident electrons and the proton target. Given that in QM the term scattering cross section denotes the probability that a particular nuclear reaction will take place, the interpretation of the flux ratio of the electrons in a scattering process as the differential scattering cross section requires an appeal to the theoretical framework of QM at a very fundamental level. ${ }^{73}$

Moreover, the data analysis in the DIS experiments was accomplished by performing "radiative corrections" on the cross section data. ${ }^{74}$ Since the radiative effects would contribute to the measured cross section at a fixed energy and angle, in scattering experiments involving charged particles radiative corrections must be made in order to eliminate the effects of the radiation of photons by electrons. In the case of the DIS experiments, radiative corrections were performed by taking into account different ways in which an electron might emit and absorb photons during its interaction with proton. To this effect, by appealing to QED, Feynman diagrams were drawn for each case, and by using these diagrams, contributions coming from the interaction of the electron with photon were calculated. In light of these contributions, the measured cross section data were revised so as to yield only the contribution coming from the interaction of electron with proton. ${ }^{75}$

Therefore, it is clear that both the data acquisition stage and the data analysis stage in the DIS experiments at SLAC were performed by making use of various theoretical considerations from various theories of HEP. ${ }^{76}$ However, since these theoretical considerations were restricted to individual stages of experimentation, like the consideration of Bjorken's scaling hypothesis, they did not continuously regulate the successive stages of the DIS experiments so as to render the experimental process theory-driven in the sense of TDE.

\subsection{The Aftermath of the Early DIS Experiments: Feynman's Parton Model, Asymptotic Freedom, and the Emergence of QCD}

The early DIS experiments at SLAC ended up with a puzzling situation; pointing to both the weak momentum transfer dependence and scaling of the deep-inelastic electron-proton scattering cross section. The preliminary results were presented in the $14^{\text {th }}$ International Conference on High Energy Physics (Vienna, August 28-September 5, 1968). In a plenary session of this conference, in his rapporteur's talk, Wolfgang Panofsky-the director of the SLAC at that

\footnotetext{
${ }^{73}$ See Friedman 1991, footnote 2, to see what kind of cross section formulation was used.

${ }^{74}$ The radiative corrections of a measured cross section belong to the perturbation expansion of QED, which is a standard tool of particle physics. The radiation correction procedures applied in the DIS experiments are discussed in more detail in Friedman and Kendall 1972, 207-209.

${ }^{75}$ Here, it is worth noting that the elimination of radiative effects occurring during the course of collision nicely illustrates the use of what Franklin calls "the Sherlock Holmes strategy", which amounts to eliminating plausible sources of error and alternative explanations of the experimental results. See Franklin 1990, 109.

${ }^{76}$ It is to be noted that theory-ladenness also arises due to the use of accelerator and detectors in particle physics experiments; because a lot of theoretical considerations, e.g. from Maxwell electrodynamics and QM, are involved in the construction of those devices. For accelerator and detector physics, see respectively Wiedemann 2007 and Green 2000. See also Hon 2003, Chalmers 2003; and Radder 2003 for a discussion of theory-ladenness due to instrumentation.
} 
time $^{77}$ - mentioned the scaling feature of the experimental data and suggested that "theoretical speculations [were] focused on the possibility that [deep-inelastic electron-proton scattering] data might give evidence on the behavior of point like, charged structures within the nucleon" (Panofsky 1968, 30). However, this was not the official view of the MIT-SLAC collaboration; as no one in the collaboration at that time had a clear explanation regarding their findings. In his Nobel Lecture, Friedman quotes the above words of Panofsky and states that the view expressed by him "was not the prevailing point of view. Even if one had proposed a constituent model at that time it was not clear that there were reasonable candidates for the constituents" (Friedman 1991, 616). ${ }^{78}$ Furthermore, the following words of Friedman clearly demonstrate that at the time of the conference the MIT-SLAC collaboration had not yet come to any definite conclusion about the findings of the DIS experiments:

[In a parallel session of the same conference,] I gave the first presentation [(Bloom et al. 1968)] of our results at the International High Energy Conference in Vienna in 1968 ... and our group had a long debate before I left about what I should say. I was instructed that under no circumstances should I talk about the possibility of pointlike structure within the proton. The general consensus was that this was too bizarre an idea to discuss in public. So I didn't. ${ }^{79}$ I showed all the data [about the scaling feature and weak momentum transfer dependence of cross section] ... but never said a word about the possibility of pointlike constituents.

Wolfgang Panofsky gave the plenary talk for that session. He said that there was experimental evidence that suggested the possibility of pointlike structure in the proton. He said it in about two sentences. But the audience appeared to pay little attention to it. The community was totally unreceptive to the idea of pointlike structure in any hadron and "Nuclear Democracy" was well entrenched in those days. (Friedman 2012, 477)

In addition to the indecisiveness of the MIT-SLAC collaboration, the above passage also indicates that, at the time of the Vienna Conference, the HEP community was not ready yet to break away from the view that suggested that the nucleon had a diffuse internal structure. In what follows, I shall outline the attempts by the theoretical HEP physicists to account for the puzzling results of the DIS experiments as well as the responses of the MIT-SLAC collaboration to these attempts.

In the late August of 1968, the Nobel Prize winner Caltech physicist Richard Feynman, who had been trying to understand hadron-hadron interactions since the mid-1960s, made a visit to the SLAC. At that time, Feynman held the belief that hadrons were composed of what he called "partons" which he conceived of as point-like structures. The inelastic electron-proton scattering data strengthened his belief in regard to the existence of partons; in the sense that in his view both the weak momentum transfer dependence and scaling observed in the DIS

\footnotetext{
${ }^{77}$ We learn from an interview, made by Harvey Lynch (April 11 and May 2, 1997), that after the elastic part of the SLAC experiments, Panofsky withdrew from the collaboration. In this interview, Panofsky said: "I was personally much involved in the optics of the magnets and I was personally involved in the elastic scattering data taking and analysis, and at that point I gave up because I felt it was unfair to younger and hard working characters who were putting their whole lives into it, so I basically backed out. This was a reasonably conscious decision" (Lynch 1997).

${ }^{78}$ In a personal communication (March 20, 2012), Bjorken said that "within the experimental group there was a spectrum of opinions at the time of the Vienna conference".

${ }^{79}$ See also Taylor 2001, 235.
} 
experiments could be explained by appealing to constituents which he called partons. Feynman conjectured that high energy hadronic collisions took place between point-like partons and that the interactions among partons were negligibly small; meaning that partons were envisaged to act as "independent" entities during the deep-inelastic scattering of electrons from the proton target (see Feynman 1969a, 1969b).

Within the framework of his parton model, Feynman found a plausible way of explaining both the scaling and the weak momentum transfer dependence of the cross section data.

Feynman regarded the scattering of electron from proton as a process during which the incoming electron emits a photon which then interacts with one of the individual partons inside the proton. Feynman's explanation of the weak momentum transfer dependence rested on an analogy that he drew from the electron-electron scattering. Due to their point-like structures, electrons typically scatter from each other at large angles. According to Feynman's view, during the scattering between the incoming electrons and the proton target the actual scattering occurs between the individual electrons and partons which were thought to be point-like structures inside the proton. Therefore, according to Feynman's parton model, electron-parton scattering had to take place at large angles; thereby explaining why at larges scattering angles scattering cross section did not fall off rapidly in the deep inelastic electron-proton scattering. In addition, Feynman showed that when each parton was regarded as the carrier of a fraction of the total momentum of the proton, i.e., $P_{p} \quad a_{\bar{r}} \quad x_{t} P_{p o} \quad r$, as depicted in Figure 4, then $x$ here could be identified as $x=1 / \omega$, where $\omega$ was the scaling parameter in Bjorken scaling. ${ }^{80}$ Thus, the phenomenon of scaling observed in inelastic scattering of electrons from protons was shown to be a natural consequence of conceiving of protons as being composed of point-like structures and carriers of the total momentum of the proton. ${ }^{81}$ In this way, it was understood that the scaling and weak momentum transfer dependence of the deep-inelastic electron-proton scattering data were essentially the same features of the scattering of electrons with point-like particles inside the proton. ${ }^{82}$

\footnotetext{
${ }^{80}$ The parton model originally developed by Feynman was soon elaborated in Bjorken and Paschos 1969, which is a follow up of Bjorken 1969. It is evident from these papers that there had been an exchange of views between Bjorken and Feynman about the formulation of the parton model during Feynman's SLAC visit.

${ }^{81}$ For more details on Feynman's parton model, see Cao 2010, sec. 6.1.

${ }^{82}$ It is to be noted that the connection between the scaling feature of deep-inelastic electron-proton scattering and the possibility of the existence of pointlike structures inside the proton was first drawn by Bjorken in an unpublished and incomplete manuscript; see Bjorken 1967a. This connection was less explicit in his publications prior to Bjorken and Paschos 1969. As we shall see, this was largely due his lack of confidence in the validity of the scaling hypothesis.
} 


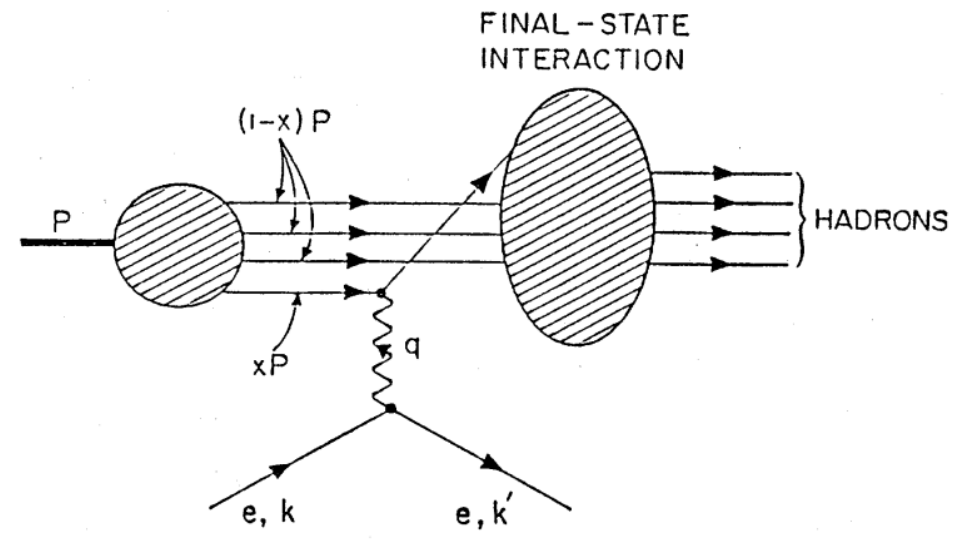

Figure 4: The deep-inelastic electron-proton scattering in the parton model. $k$ and $k^{\prime}$ represent the incident and final momentum of the electron, respectively, $\mathrm{q}$ stands for the photon exchanged during the scattering, and $\mathrm{x}$ is a certain fraction of the proton's momentum. From Bjorken and Paschos 1969.

At around the same time, drawing on Bjorken's formulation of scaling, in a jointly written paper, the American physicists Curtis Callan and David Gross showed that for large momentum transfer the ratio $R=\sigma_{L} / \sigma_{l}$, where $\sigma_{L}$ and $\sigma_{T}$ represent respectively the cross sections for longitudinal and transverse polarized virtual photons exchanged between partons and electrons, depended heavily on the spin of the constituents of the nucleons (see Callan and Gross 1969). Moreover, they showed that the vanishing of $R$ in the limit where both $q^{2}$ and $v$ become sufficiently large, i.e., in the deep-inelastic scattering regime, would imply that the nucleon was made only out of spin-1/2 particles as suggested by the quark model; whereas the non-vanishing of $R$ in the same limit would imply that the constituents of the nucleon included spin- 0 or spin-1 particles. Upon this result, Jeffrey Mandula specified the kinematic variation of $R$ that was required by the scaling hypothesis; he showed that $R$ should diminish like $1 / v$ in the deep-inelastic scattering regime (Mandula1973). The experimental confirmation of these theoretical predictions arrived quickly. In the $15^{\text {th }}$ International Conference on High-Energy Physics (Kiev, August 26September 4, 1970), the MIT-SLAC collaboration presented the data showing that $R=0.18 \pm 0.5$ (see Bloom et al. 1970). ${ }^{83}$ As we learn from Friedman's Nobel Lecture, "[b]y the time of the [Kiev Conference the members of the MIT-SLAC collaboration] were reasonably convinced that [they] were seeing constituent structure in [their] experimental results " (Friedman 1991, 623). In the subsequent experiments, $R$ was measured over a larger kinematic range and its accuracy was sufficiently improved by decreasing the errors (see Miller et al. 1972; and Riordan et al. 1974). Furthermore, it was shown that, for $\omega \leq 5$, the kinematic variation of $R$ was consistent with scaling, i.e., it behaved as $1 / v$ in the deep-inelastic scattering regime as previously suggested by Mandula. The MIT-SLAC collaboration interpreted these results as indicating the existence of spin- $1 / 2$ constituents according to the parton model of the nucleon. ${ }^{84}$

\footnotetext{
${ }^{83}$ Note that a preliminary analysis of the data had been reported by Taylor (1970) at the 4th International Symposium on Electron and Photon Interactions at High Energy held in Liverpool between September 14 and 20 in 1969.

${ }^{84}$ See the abstract of Riordan et al. 1974. Note that the parton model does not single out a specific spin value for the constituents of the nucleon.
} 
The experimental results that came out from the improved DIS experiments urged the theoretical HEP community to study more closely the scaling hypothesis in relation to the possibility that the nucleon consisted of spin-1/2 point-like constituents as previously proposed by Gell-Mann and Zweig. A key step in this endeavor was the mechanism of "asymptotic freedom" that was proposed jointly by Gross and his doctoral student Franz Wilczek at Princeton University and independently by David Politzer at Harvard University (see Gross Wilczek 1973; and Politzer 1973). According to this mechanism, at high energies the interactions between the constituents of nucleons, which are today referred to as "quarks", become weak at short distances. The mechanism of asymptotic freedom was able to account for why the interactions between the constituents of the protons were negligible; a feature that had been left unexplained in Feynman's parton model. The hypothesis of asymptotic freedom opened the door to the development of a more comprehensive theory of strong interactions, namely QCD, that would subsequently replace the SMT. ${ }^{85}$

\section{Conclusions: The Exploratory Character of Experimentation}

In this paper, I have introduced a categorization of the theories of HEP — as background theory, model theory and phenomenological model - based on the ways in which they relate to subatomic phenomena. Drawing on this categorization, I have contrasted the distinct roles played by theoretical considerations and distinguished between the weak and strong senses of TLE in the context of two cases of experimentation from the history of HEP: namely, the elastic protonproton scattering experiments of the first half of the 1960s and the DIS experiments at SLAC between the years of 1967 and 1973. I have argued that in the former case each stage of experimentation, from initial planning and design up to data analysis and interpretation, was carried out by appealing to a particular phenomenological model of HEP, namely the Regge theory, for the sole purpose of testing the conclusions of this theory. I have argued that this case illustrates the strong sense of TLE, which I have previously called TDE. Note that the latter reflects what the theory-dominated view of scientific experimentation suggests as the only type of relationship between theory and experiment in scientific practice. Here, I by no means draw a necessary link between theory-driven experimentation and phenomenological models of HEP such that all theory-driven experiments in HEP are carried out by means of phenomenological models. But, I suggest that given their capability of offering more detailed accounts of subatomic phenomena as well as directly testable predictions, it is typical that theory-driven experiments in HEP are carried out by means of phenomenological models. By contrast, in the case of the DIS experiments at SLAC, experimentation lacked the guidance of any phenomenological model of the target phenomena. As a result, the experimental stages were not continuously regulated under the guidance of any theoretical account; indicating that experimentation was carried out to a great extent autonomously. Even though certain theories were relied upon during experimentation, the influence of these theories on the overall progress of experimentation remained local and minimal, in that their use was limited to certain stages of experimentation and provided only the most basic instrumental and conceptual requirements to perform experimentation in HEP. These included relying on QM and Maxwell's electrodynamics

\footnotetext{
${ }^{85}$ Interested readers will find more information in Cao 2010, section 6.1 about the parton model, and 8.1 about asymptotic freedom. For the construction of QCD, see Cao 2010, chaps. 7 and 8; and Pickering 1984, chap. 7. Also, for the fate of the SMRP, see Cushing 1990, sec. 6, where it is argued that by the 1970s the SMRP had become a canonical example of a Lakatosian "degenerating research program."
} 
to build and use particle detectors to carry out scattering cross section measurements, as well as relying on QED to perform radiative corrections to the measured scattering cross section data. Therefore, I conclude that the DIS experiments at SLAC illustrate what I have previously called the weak sense of TLE.

The theory-dominated view of scientific experimentation acknowledges the testing of the conclusions of scientific theories and hypotheses as the only role experimentation can play in scientific practice. However, in the case of the DIS experiments this view is far less plausible. Even though Bjorken's prediction of a large scattering cross section for backward inelastic scattering of electrons from nucleons was available prior to these experiments, the experimentalists' narratives suggest that, for the reasons I have stated in the preceding discussion, the testing of Bjorken's result was not originally part of their agenda. Therefore, the fact that there exists a theoretical account of the phenomena to be studied in an experiment does not entail that it would be taken into account by the experimentalists. In the end, it is experimentalists' considerations and decisions that shape the scope and content of the agenda to be pursued in an experiment; suggesting that it would be descriptively inaccurate to simply assume that the theorists point out to the experimentalists what phenomena to study in an experiment. These considerations show how experiments actually get their own life as previously suggested by Hacking, and they lend support to the view that experimentation enjoys a certain amount of autonomy from "theory", as earlier suggested by Galison.

The above discussion suggests that even though in both historical cases I have analyzed in this paper experimentation is theory-laden, the senses of TLE that are at work are qualitatively different. Therefore, in the history of HEP "theory" plays qualitatively different roles in experimentation and that this in turn calls for a differentiation of different degrees, and thus different senses, of TLE. From this, I draw the conclusion that TLE should be understood as an umbrella concept that has varying senses. This conclusion stands in contrast with the theorydominated view of scientific experimentation that takes the concept of TLE undifferentiated and that treats all relations of theorizing and experimentation on a par; namely, that experiments in science are performed solely to ascertain the conclusions of scientific theories or hypotheses.

If the DIS experiments do not play the role of testing, what other role do they suggest for experimentation in the history of HEP? I shall argue that these experiments illustrate an exploratory use of experimentation in the practice of HEP and thereby suggest the role of exploration for experimentation. Here, in a similar vein to Steinle, I use the term "exploratory" to characterize the type of experimentation that is performed without having recourse to any theoretical account of its target phenomena. I shall characterize the exploratory character of the DIS experiments in terms of the following aspects: the general aim of experimental inquiry; the experimental methods or strategies used; and the involvement of theoretical considerations in experimentation. In what follows, I shall consider these aspects of the DIS experiments and argue that it was the combination of those aspects that lent the DIS experiments their exploratory character. $^{86}$

To this end, I shall first note that the DIS experiments were carried out with an explorative aim; in that they undertook to probe a scattering regime that had not yet been probed by previous experiments and about which there was no well-defined and well-established

\footnotetext{
${ }^{86}$ Here, I follow Elliott's account that suggests that exploratory experimentation should be characterized along three dimensions: the positive aim of the experimental activity; the methods or strategies used for varying parameters; and the role that theory plays in the experimental activity (Elliott 2007).
} 
theoretical framework. ${ }^{87}$ In this respect, the DIS experiments were carried out not to test any theoretical account, but rather to advance the understanding of the inner structure of the nucleon through the study of an as-yet-unexplored scattering regime. With respect to the experimental method used in the DIS experiments, I shall note that the finding of the weak momentum transfer dependence of the deep-inelastic electron-proton scattering cross section was obtained not through the guidance of a particular theory or model but rather as a result of the decision of the systematic investigation of an as-yet-unexplored scattering regime. This led to the success of the DIS experiments; if only the forward scattering region had been probed in these experiments, none of their findings would have been obtained. The experimental strategy adopted in the DIS experiments was to systematically vary the experimental parameters so as to allow the probing of both the forward and backward scattering regions. ${ }^{88}$ More specifically, the scattering cross section was measured at different scattering angles (corresponding to different momentum transfer values) at different energies, as indicated in Figure 3. Note that this experimental strategy allows searching for any possible regularities that might exist in the scattering spectrum, as opposed to only one specific effect already predicted by some theoretical account. In this respect, the experimental strategy adopted in the DIS experiments was not biased towards or against the detection of a previously predicted effect. Lastly, with respect to the involvement of theoretical considerations in experimentation, I shall note that the DIS experiments were performed in a way largely autonomous from "theory", as I have previously argued. Thus, the above considerations point to the exploratory character of the DIS experiments in that they were performed to fulfill an exploratory objective by adopting a suitable exploratory strategy that was implemented in a way largely autonomous from "theory". Note that this last point suggests that the exploratory character of the DIS experiments is closely linked to their autonomy from "theory". This also receives support from the following quotation by Gross:

Early scattering experiments concentrated, for obvious reasons, on the events that had the largest rates. In the case of the strong interactions, this meant searching for resonant bumps or probing near forward scattering, where the cross section was largest. It was not at all realized by theorists that the secret of hadronic dynamics could be revealed by experiments at large momentum transfer that probed the short distance structure of hadrons ... Therefore theorists concluded that Regge behavior must be very important and forward scattering experiments were deemed to be the major tool of discovery. Regge theory was soon incorporated into the bootstrap program as a boundary condition. In response to this theoretical enthusiasm, the interest of experimentalists in forward scattering was enhanced. Opportunities to probe the less easily accessible domains of large momentum transfer were ignored. Only much later, after the impact of the deepinelastic scattering experiments that had been ridiculed by many as unpromising, was it understood that the most informative experiments were those at large

\footnotetext{
${ }^{87}$ Note that, as previously mentioned, prior to the DIS experiments the lower bound obtained by Bjorken on the cross section of inelastic backward electron-nucleon scattering was the only relevant theoretical result available. However, as the later theoretical developments showed, this result by itself was not conclusive either about the inner structure of the nucleon or the deep-inelastic scattering regime. Nor was it a well-established theoretical result; as mentioned earlier, even Bjorken himself did not feel confident enough at that time about the correctness and implications of this result. Similarly, elsewhere Bjorken noted: "While I now look back with considerable satisfaction at all this, I most emphatically add that at the time I didn't have much confidence in what was basically a lot of guesswork. Was this in fact legitimate theoretical physics? It was not clear at all" (Bjorken 1997, 593)

${ }^{88}$ Note that this experimental strategy illustrates one of the exploratory experimental strategies Steinle speaks of.
} 
momentum transfers that probe short ... distances. (Gross 2004, 197-198)

When evaluated in terms of their outcomes, the DIS experiments can be said to have had significant impact on the development of the subsequent research agenda in theoretical HEP. Particularly, the findings of the DIS experiments (namely, the weak momentum transfer dependence and scaling of the scattering cross section data) were in conflict with the widely held view of the inner structure of the nucleon; namely that nucleons had a diffuse inner structure and could not serve to diffract impinging electrons at large angles corresponding to large momentum transfer during scattering. The findings of the DIS experiments put this view of the nucleon into question and thus lent great impetus to the theoretical study of the short-distance behavior of the nucleon that was previously largely neglected by physicists due to their commitments to a particular view. Although Bjorken put forward the scaling hypothesis before the DIS experiments, only after it was used in the formulation of the weak momentum transfer dependence of the SLAC deep-inelastic scattering cross-section data did the scaling hypothesis become thoroughly discussed and appreciated within the HEP community. As the following passage indicates, even Bjorken himself felt precarious about the validity of the scaling hypothesis during the period before all the deep inelastic scattering cross section data were available:

[Prior to the DIS experiments] ... I had a pretty good idea of the scaling hypothesis and its connection with a pointlike-constituent interpretation. But what comes through with less clarity was my lack of confidence in its correctness. It was one of several options regarding how the experiments would turn out. And I did not fully commit to the pointlike-constituent view until all the returns were in." 89

The scaling hypothesis should be seen as the first concrete attempt toward a novel account of the nucleon. However, as the above passage seems to indicate, even in the eyes of its founder it was a precarious attempt when it was first proposed. In early seventies, as the results of the DIS experiments started to gain the trust of the theoretical physicists, the scaling hypothesis started to attract the attention of the theoretical HEP community; as it was the only theoretical account that explained the results of the DIS experiments. The following quotation by Gross exemplifies the impact of the results of the DIS experiments on the works of the theoretical physicists who had been trying to understand the inner structure of the nucleon:

SLAC deep-inelastic scattering experiments had a profound impact on me. They clearly showed that the proton behaved, when observed over short times, as if it were made out of pointlike objects of spin one-half. (Gross 2004, 199)

The concept of asymptotic freedom was soon proposed to provide the concept of scaling with a quantum-field theoretic description, which was missing in Feynman's parton model. ${ }^{90}$ The above discussion indicates that the DIS experiments not only triggered but also constituted the driving

\footnotetext{
${ }^{89}$ Bjorken (personal communication, March 20, 2012).

${ }^{90}$ This was clearly indicated in the opening paragraph of Gross and Wilczek 1973, 1343, as follows: "We therefore suggest that one should look to a non-Abelian gauge theory of the strong interactions to provide the explanation for Bjorken scaling, which has so far eluded field theoretic understanding".
} 
force behind the historical process through which novel concepts of the inner structure of the nucleon and novel accounts based on those concepts were developed.

This conclusion also lends support to Steinle's claim that exploratory experimentation has the potential not only to bring about the revision of the existing concepts and theoretical frameworks, but also to contribute to the formation of novel theoretical frameworks in which novel concepts can be used. Moreover, the explanation of weak momentum transfer dependence of the deep-inelastic electron-proton scattering cross section through the concept of scaling in Feynman's parton model as well as the fact that the scaling behavior of the inelastic cross section data was experimentally discovered following Bjorken's scaling hypothesis indicate that theoretical considerations are essential for interpreting experimental data as well as for situating the experimental results in a theoretical context. All these considerations point to a time period of mutual interaction and cooperation between theory and experiment, where the question of which one comes first turns out to be ill-posed.

Consequently, the present case study on the DIS experiments suggests the lesson that there is room for experimentation to be exploratory while being at the same time theory-laden due to the theoretical context provided by well-established theories occupying the research domain of interest. Hence, the exploratory character of scientific experimentation as well as the epistemic appropriateness of the term "exploratory experimentation" are not restricted to research fields lacking a well-established theoretical framework-such as the ones discussed previously by Steinle and others - but they are also valid in research domains which are occupied by well-established theories, or so to speak, mature theories of science. Another important difference between the present case study and Steinle's case studies concerns the "division of labor" in scientific research. In Steinle's case studies, the experimentalists who conducted exploratory experiments were also the ones who developed novel concepts to account for the findings of these experiments. However, in the present case study, there is a clear distinction between the group of experimentalists and the group of theorists who formulated the findings of these experiments on the basis of novel concepts. Therefore, the difference between the present case study and Steinle's case studies stems from the fact that while the latter concerns the early period of a research domain, namely static electricity, where there was yet no professional specialization, the former concerns a research domain, namely HEP, that achieved a high degree of professional specialization that resulted in a separation among the groups of experimentalists and theorists. I take the last point to indicate the changing face of exploratory experimentation in the practice of science.

\section{Acknowledgments}

This work would not have been possible without the guidance and encouragement of Jutta Schickore. I am highly indebted to her for introducing me to the history and philosophy of scientific experimentation. I would also like to thank Nick Best, James Bjorken, Jerome Friedman, Hans Radder, Friedrich Steinle, Christian Zeitnitz as well as two anonymous referees and the editors of this journal for reading and providing helpful suggestions on earlier versions of this paper. I also benefited from discussions with Arianna Borrelli, Jordi Cat, Allan Franklin, Amit Hagar, Efe Yazgan and the audiences at The First Conference on Integrated History and Philosophy of Science (The University of Pittsburgh, USA, 11-14 October 2007) and The $24^{\text {th }}$ Boulder Conference in History and Philosophy of Science (The University of Colorado, USA, October 10-12, 2008), where earlier versions of this paper were presented. 


\section{References}

Ackermann, Robert. 1989. "The New Experimentalism." British Journal for the Philosophy of Science 40:185-190.

Adler, Stephen.1966. "Sum Rules Giving Tests of Local Current Commutation Relations in High Energy Neutrino Reactions." Physical Review 143:1144-1155.

Albert, David Z. 1994. Quantum Mechanics and Experience. Cambridge: Harvard University Press.

Auyang, Sunny Y. 1995. How is Quantum Field Theory Possible. New York: Oxford University Press.

Binosi Daniele and Theussl, Lukas. 2004. "JaxoDraw: A Graphical User Interface for Drawing Feynman Diagrams." Computer Physics Communications 161:76-86.

Bjorken, James D. 1966a. "Inequality for Electron and Muon Scattering from Nucleons." Physical Review Letters 16:408.

Bjorken, James D. 1966b. "Applications of the Chiral U(6)®U(6) Algebra of Current Densities." Physical Review 148:1467-1478.

Bjorken, James D. 1967a. "Inelastic Electron (and Muon) Scattering at High Energies and Forward Angles". An incomplete, unpublished manuscript written in early 1967; a refined version published in Bjorken 2003, 27-39, under the title "Inelastic Lepton Scattering and Nucleon Structure".

Bjorken, James D. 1967b. "Current Algebra in Small Distances." In Proceedings of International School of Physics, Enrico Fermi Course 41, Selected Topics in Particle Physics (Varenna, July 17-29, 1967), edited by Jack Steinberger, 55-81. London and New York: Academic Press.

Bjorken, James D. 1967c. "Theoretical Ideas on Inelastic Electron and Muon Scattering", in Proceedings of the International Symposium on Electron and Photon Interactions at High Energies (Stanford University, September 5-9, 1967), edited by Berman M. Samuel, 109-127. Springfield: International Union of Pure and Applied Physics.

Bjorken, James D. 1967d. "Inequality for Backward Electron- and Muon-Nucleon Scattering at High Momentum Transfer." Physical Review 163:1767-1769.

Bjorken, James D. 1969a. "Theoretical Ideas on High-Energy Inelastic Electron-Proton Scattering", paper delivered at the American Physical Society Meeting (New York, February 3, 1969). SLAC Pre-print: SLAC-PUB-571. Re-printed in Bjorken 2003, 41-52.

Bjorken, James D. 1969b. “Asymptotic Sum Rules at Infinite Momentum.” Physical Review 179:1547-1553 
Bjorken, James D. 1997. "Deep-Inelastic Scattering: From Current Algebra to Partons." In The Rise of the Standard Model: Particle Physics in the 1960s and 1970s, edited by Hoddeson, Lillian, Laurie M. Brown, Michael Riordan and Max Dresden, 589-599. Cambridge: Cambridge University Press.

Bjorken, James D., 2003. In Conclusion: A Collection of Summary Talks in High Energy Physics. Singapore: World Scientific.

Bjorken James and Paschos Emmanual A. 1969. "Inelastic Electron-Proton and $\gamma$-Proton Scattering and the Structure of the Nucleon." Physical Review 185:1975-1982.

Bloom, Elliott D., et al. 1968. "Inelastic Scattering from Protons." Not published but listed as paper 563 on page 456 in Proceedings of 14th International Conference on High Energy Physics (Vienna, August 28-September 5, 1968), edited by Jack Steinberger and Jacek Prentki. Geneva: European Organization for Nuclear Research.

Bloom, Elliott D., et al. 1969. "High-Energy Inelastic e-p Scattering at $6^{\circ}$ and $10^{\circ}$. . Physical Review Letters 23:930-934.

Bloom, Elliott D., et al. 1970. "Recent Results in Inelastic Electron Scattering." Unpublished report presented to the $15^{\text {th }}$ International Conference on High Energy Physics, Kiev, U.S.S.R, August 26-September 4, 1970. SLAC-Pre-print: SLAC-PUB-796.

Breidenbach, Martin et al. 1969. "Observed Behaviour of Highly Inelastic Electron-Proton Scattering." Physical Review Letters 23:935-939.

Brown, Harvey R. and Harré Rom, eds. 1988. Philosophical Foundations of Quantum Field Theory. Oxford: Clarendon Press.

Burian, Richard M. 1997. "Exploratory Experimentation and the Role of Histochemical Techniques in the Work of Jean Brachet, 1938-1952." History and Philosophy of the Life Sciences 19:27-45.

Burian, Richard M. 2007. "On microRNA and the Need for Exploratory Experimentation in Post-Genomic Molecular Biology." History and Philosophy of the Life Sciences 29(3):285-311

Callan, Curtis G. and Gross, David J. 1969. "High-Energy Electroproduction and the Constitution of Electric Current." Physical Review Letters 22:156.

Cao, Tian Y. 1991. "Attempts at Reconciling Quantum Field Theory with S-matrix Theory -The Reggeization Program: 1962-82." The Archive for History of Exact Sciences 41:239-83.

Cao, Tian Y. 1997. Conceptual Developments of Twentieth Century Field Theories. Cambridge: Cambridge University Press. 
Cao, Tian Y., ed. 1999. Conceptual Foundations of Quantum Field Theory, Cambridge University Press.

Cao, Tian Y. 2010. From Current Algebra to Quantum Chromodynamics: A Case for Structural Realism. Cambridge: Cambridge University Press.

Carrier, Martin. 1998. "New Experimentalism and Changing Significance of Experiments. On the Shortcomings of an Equipment-Centered Guide to History." In Experimental Essays-Versuche zum Experiment, edited by Michael Heidelberger and Friedrich Steinle, 175191. Baden-Baden: Nomos.

Cartwright, Nancy. 1983. How the Laws of Physics Lie. Oxford: Clarendon Press.

Cartwright, Nancy, Towfic Shomar and Mauricio Suarez. 1995. "The Tool Box of Science:

Tools for the Building of Models with a Superconductivity Example." In Theories and Models in Scientific Process (Poznan Studies in the Philosophy of Science and the Humanities 44), edited by Herfel, William, Wladiyslaw Krajewski, Ilkka Niiniluoto and Ryszard Wojcicki, 137-149. Amsterdam: Rodopi.

Chalmers, Alan. 2003. "The Theory-Dependence of the Use of Instruments in Science." Philosophy of Science 70:493-509.

Chew, Geoffrey F. 1961. S-Matrix Theory of Strong Interactions. New York: Benjamin Inc.

Chew, Geoffrey F. 1962. "The S-matrix Theory of Strong Interactions without Elementary Particles." Review of Modern Physics 34:394-401.

Chew, Geoffrey F. 1963. "Strong Interaction S-Matrix Theory Without Elementary Particles." In 1962 Cargese Lectures in Theoretical Physics, edited by Maurice Levy, chap. 11. New York: Benjamin Inc.

Chew, Geoffrey F. 1964. "Nuclear Democracy and Bootstrap Dynamics". In Strong-Interaction Physics: A Lecture Note Volume, edited by Maurice Jacob and Geoffrey F. Chew, 103-152. New York: Benjamin.

Chew, Geoffrey F. 1968. “ “Bootstrap': A Scientific Idea.” Science 161:762-765.

Chew, Geoffrey F. 1970. “Hadron Bootstrap: Triumph or Frustration?” Physics Today 23:23-28.

Chew, Geoffrey F. 1989. "Particles as S-matrix Poles: Hadron Democracy". In Pions to Quarks: Particle Physics in The 1950s, edited by Brown, Laurie M., Max Dresden and Lillian Hoddeson, 600-608. Cambridge: Cambridge University Press.

Chew, Geoffrey F. and Frautschi, Steven C. 1961a. "Principle of Equivalence for All Strongly Interacting Particles within the S-matrix Framework.” Physical Review Letters 7:394-397. 
Chew, Geoffrey F. and Frautschi, Steven.1961b. "Dynamical Theory for Strong Interactions at Low Momentum Transfers But Arbitrary Energies.” Physical Review 123:1478-1486.

Chew, Geoffrey F., Steven Frautschi and Stanley Mandelstam. 1962. "Regge Poles in Pion-Pion Scattering." Physical Review 126:1202-1208.

Chew, Geoffrey F., Murray Gell-Mann and Arthur H. Rosenfeld. 1964. "Strongly Interacting Particles." Scientific American 210:74-93.

Clifton, Robert K., ed. 1996. Perspectives on Quantum Reality: Non-relativistic, Relativistic, and Field-theoretic. Boston: Kluwer Academic Publishers

Collins, Peter D. B. 1977: An introduction to Regge Theory and High-Energy Physics. Cambridge: Cambridge University Press.

Cobb, Aaron D. 2009. "Michael Faraday's "Historical Sketch of Electro-Magnetism” and the Theory-Dependence of Experimentation.” Philosophy of Science 76:624-636.

Coward, David H. et al. 1968. "Electron-Proton Elastic Scattering at High Momentum Transfers." Physical Review Letters 20:292-295.

Cushing, James T. 1985. "Is There Just One Possible World? Contingency vs the Bootstrap." Studies in History and Philosophy of Science 16:31-48.

Cushing, James T. 1990. Theory Construction and Selection in Modern Physics: The S-matrix. Cambridge: Cambridge University Press.

Diddens, Albert N. et al.1962. "High Energy Proton-Proton Scattering." Physical Review Letters 9:111-114.

Drell, Sidney D and Walecka, John D. 1964. "Electrodynamics Processes with Nuclear Targets." Annals of Physics 28:18-33.

Duhem, Pierre M. M. 1991: The Aim and Structure of Physical Theory. Translated from the French by Philip P. Wiener. Princeton: Princeton University Press.

Elliott, Kevin C. 2007. "Varieties of Exploratory Experimentation in Nanotoxicology." History and Philosophy of the Life Sciences 29:311-334.

Falkenburg, Brigitte. 2007. Particle Metaphysics: A Critical Account of Subatomic Reality. Berlin Heidelberg: Springer.

Feynman, Richard P. 1969a: "Very High-Energy Collisions of Hadrons." Physical Review Letters 23:1415-1417. 
Feynman, Richard P. 1969b. "The Behavior of Hadron Collisions at Extreme Energies.” In Proceedings of the $3^{\text {rd }}$ International Conference on High Energy Collisions, edited by Chen N. Yang et al., 237-256. New York: Gordon and Breach.

Feynman, Richard P. 1972. Photon-Hadron Interactions, Reading, Mass.: W. A. Benjamin.

Feyerabend, Paul. 1981. Realism, Rationalism, and Scientific Method, Philosophical Papers, vol. I. Cambridge: Cambridge University Press.

Foley, Kenneth J. et al. 1963. "7- to $20-\mathrm{BeV} / c \pi^{-}+p$ and $p+p$ Elastic Scattering and Regge Pole Predictions." Physical Review Letters 10:376-381.

Franklin, Allan. 1986. The Neglect of Experiment. Cambridge: Cambridge University Press.

Franklin, Allan. 1990. Experiment, Right or Wrong. Cambridge: Cambridge University Press.

Franklin, Allan. 1997. “Millikan’s Oil-drop Experiments.” The Chemical Educator 2:1-14.

Franklin, Laura. 2005. “Exploratory Experiments.” Philosophy of Science (Proceedings) 72:888899.

Freundlich, Yehudah. 1980. "Theory Evaluation and the Bootstrap Hypothesis.” Studies in History and Philosophy of Modern Physics 4:267-277.

Frautschi, Steven. C., Murray Gell-Mann and Fredrik Zachariasen. 1962. "Experimental Consequences of the Hypothesis of Regge Poles.” Physical Review 126:2204-2218.

Friedman, Jerome I. and Kendall, Henry W. 1972. "Deep Inelastic Electron Scattering.” Annual Review of Nuclear Science 22:203-254.

Friedman, Jerome I. 1991. "Deep-inelastic Scattering: Comparisons with the Quark Model." Nobel Prize Lecture delivered 8 December 1990, Review of Modern Physics 63:615-627.

Friedman, Jerome I. 2012. "Peering Inside the Proton." European Physical Physical Journal H, 36:469-485.

Fujii, Tadao et al. 1962. "Elastic Proton-Proton Scattering at 1.35, 2.1, and 2.9 BeV." Physical Reiew 128:1836-1841.

Gale, George, 1974. “Chew’s Monadology.” Journal of the History of Ideas 35:339-348.

Galison, Peter. 1987. How Experiments End. Chicago: University of Chicago Press.

Galison, Peter. 1997. Image and Logic: A Material Culture of Microphysics. Chicago: University of Chicago Press. 
Gell-Mann, Murray.1962. "Symmetries of Baryons and Mesons.” Physical Review 125:1067 1084.

Gell-Mann, Murray. 1964. “A Schematic Model of Baryons and Mesons.” Physics Letters 8:214215.

Giere, Ronald. 1988. Explaining Science. A Cognitive Approach. Chicago: University of Chicago Press.

Gooding, David. 1990. Experiment and the Making of Meaning: Human Agency in Scientific Observation and Experiment. Dordrecht; Boston: Kluwer Academic Publishers.

Green, Dan. 2000. The Physics of Particle Detectors. Cambridge: Cambridge University Press.

Griffiths, D., 2008. Introduction to Elementary Particles, $2^{\text {nd }}$ rev. edition. New York: Wiley

Gross, David J. and Wilczek Franz. 1973. "Ultraviolet Behavior of Non-Abelian Gauge

Theories.", Physical Review Letters, 30: 1343-1346.

Gross, David J. 2005. “Asymptotic Freedom and QCD- A Historical Perspective.” Nuclear Physics B (Proceedings Supplements) 135:193-211.

Hacking, Ian. 1983. Representing and Intervening: Introductory Topics in the Philosophy of Natural Science. Cambridge: Cambridge University Press.

Hacking, Ian. 1992. "The Self Vindication of the Laboratory Science." In Science as Practice and Culture, edited by Andrew Pickering, 29-64. Chicago: The University of Chicago Press.

Hanson, Norwood R. 1960. Patterns of Discovery. An Inquiry into the Conceptual Foundations of Science. Cambridge: Cambridge University Press.

Hartmann, Stephan. 1998. "Idealization in Quantum Field Theory." In Idealization in Contemporary Physics (Poznan Studies in the Philosophy of Science and the Humanities 64), edited Niall Shanks, 99-122. Amsterdam: Rodopi.

Hartmann, Stephan. 1999. "Models and Stories in Hadron Physics.” In Models as Mediators: Perspectives on Natural and Social Science, edited by Mary Morgan and Margaret Morrison, 326-346. Cambridge: Cambridge University Press.

Healey, Richard. 1989. The Philosophy of Quantum Mechanics: An Interactive Interpretation. Cambridge: Cambridge University Press.

Heidelberger, Michael. 2003. "Theory-Ladenness and Scientific Instruments in Experimentation.” In The Philosophy of Scientific Experimentation, edited by Hand Radder, 138151. Pittsburgh: University of Pittsburgh Press. 
Heisenberg, Werner. 1943a. "Die "Beobachtbaren Grossen” in der Theorie der Elementarteilchen. I.” Zeitschrift für Physics 120:513-538.

Heisenberg, Werner. 1943b. "Die "Beobachtbaren Grossen" in der Theorie der Elementarteilchen. II.” Zeitschrift für Physics 120:673-702.

Hofstadter, Robert. 1956. "Electron Scattering and Nuclear Structure." Review of Modern Physics 28:214-254.

Hofstadter, Robert and Robert, McAllister. 1955. "Electron Scattering from the Proton." Physical Review 98:217-218.

Hon Giora. 2003. "The idols of Experiment: Transcending the "Etc. List"." In The Philosophy of Scientific Experimentation, edited by Hand Radder, 174-97. Pittsburgh: University of Pittsburgh Press.

Hughes, R. I. G., 1992. The Structure and Interpretation of Quantum Mechanics. Cambridge, Mass.: Harvard University Press.

Jones, Lawrence W. 1977. “A Review of Quark Search Experiments.” Review of Modern Physics 49:717-752.

Kaiser, David. 2002. "Nuclear Democracy Political Engagement, Pedagogical Reform, and Particle Physics in Postwar America.” Isis 93:229-268.

Kaiser, David. 2005. Drawing Theories Apart: The Dispersion of Feynman Diagrams in Postwar Physics. Chicago: The University of Chicago Press.

Karaca, Koray. Forthcoming. "The construction of the Higgs Mechanism and the Emergence of the Electro-weak Theory." Studies in History and Philosophy of Modern Physics.

Kendall, Henry W. 1991. "Deep-inelastic Scattering: Experiments on the Proton and the Observation of Scaling." Nobel Prize Lecture delivered 8 December 1990, Review of Modern Physics 63:597-615.

Kuhn, Thomas. 1970. The Structure of Scientific Revolutions, $2^{\text {nd }}$ enlarged ed. Chicago: The University of Chicago Press.

Lynch H., 1997. Interview with Dr. Wolfgang K. H. Panofsky, at the Stanford Linear Accelerator Center, April 11 and May 2; the transcript of the interview is available at the URL: http://www.aip.org/history/ohilist/5906.html

Mandula, Jeffrey.1973. "Scaling Limit of Longitudinal Virtual Compton Cross Sections." Physical Review D 8:328-330. 
Marcum, James. 2007. “Experimental Series and the Justification of Temin's DNA Provirus Hypothesis." Synthese 154:259-292.

Miller, Gerald et al. 1972. "Inelastic Electron-Proton Scattering at Large Momentum Transfers." Physical Review D 5:528-544.

Morgan, Mary and Morrison, Margaret. 1999. "Models as Mediating Instruments." In Models as Mediators: Perspectives on Natural and Social Science, edited Mary Morgan and Margaret Morrison, 10-37. Cambridge: Cambridge University Press.

Morrison, Margaret. 1999. "Models as Autonomous Agents.” In Models as Mediators:

Perspectives on Natural and Social Science, edited by Mary Morgan and Margaret Morrison, 3865. Cambridge: Cambridge University Press.

Neal, Richard B. 1967. "Completion of Construction and Initial Operation of the SLAC Accelerator." IEEE Transactions of Nuclear Science, June.

O’Malley, Maureen. 2007. "Exploratory Experimentation and Scientific Practice: Metagenomics and the Proteorhodopsin Case." History and Philosophy of the Life Sciences 29 (3):335-358

Panofsky, Wolfgang K. H. 1968. "Electromagnetic Interactions: Low $q^{2}$ Electrodynamics: Elastic and Inelastic Electron (and Muon) Scattering." In Proceedings of the $14^{\text {th }}$ International Conference on High Energy Physics (Vienna, August 28-September 5), edited by Jacek Prentki and Jack Steinberger, 23-39. Geneva: CERN.

Pickering, Andrew. 1984. Constructing Quarks: A Sociological History of Particle Physics. Chicago: The University of Chicago Press.

Politzer, David. 1973. "Reliable Perturbative Results for Strong Interactions." Physical Review Letters 30:1346-1349.

Popper, Karl. 1959. The Logic of Scientific Discovery. London: Hutchinsion.

Radder, Hans. 2003. "Technology and Theory in Experimental Science.” In The Philosophy of Scientific Experimentation, edited by H. Radder, 152-173. Pittsburgh: University of Pittsburgh Press.

Radder, Hans. 2006. The World Observed, the World Conceived. Pittsburgh: University of Pittsburgh Press.

Rechenberg, Helmut. 1989. "The Early S-matrix Theory and its Propagation.” In Pions to Quarks: Particle Physics in The 1950s, edited by Brown, Laurie M., Max Dresden and Lillian Hoddeson, 551-578. Cambridge: Cambridge University Press.

Redhead, Michael. 1980a. "Models in Physics." British Journal for the Philosophy of Science 31:145-163. 
Redhead, Michael. 1980b. "Some Philosophical Aspects of Particle Physics." Studies in History and Philosophy of Science 11:279-304.

Redhead, Michael. 1982. "Quantum Field Theory for Philosophers.” In Proceedings of the Biennial Meeting of the Philosophy of Science Association 2:57-99.

Redhead, Michael. 2005. "Broken Bootstraps-The Rise and Fall of a Research Programme", Foundations of Physics 35:561-75.

Regge, Tullio. 1959. "Introduction to Complex Orbital Momenta." Nuovo Cimento 14:951-976.

Regge, Tullio. 1960. "Bound States, Shadow States and Mandelstam Representation.” Nuovo Cimento 18:947-956.

Riordan, Michael et al. 1974. "Extraction of Deep Inelastic e-p and e-d Cross Sections.” Physical Review Letters 33:561-564.

Riordan, Michael. 1987. The Hunting of the Quark: A True History of Modern Physics. New York: Simon \& Schuster.

Ruetsche, Laura. 2011. Interpreting Quantum Theories: The Art of the Possible. Oxford: Oxford University Press.

Sargent, Rose-Mary. 1995. "Exploratory Experiments: Scientists at Play." Unpublished manuscript of a History of Science Society Lecture.

Schweber, Silvan S. 1994. QED and the Men Who Made It: Dyson, Feynman, Schwinger, and Tomonaga. Princeton, N.J.: Princeton University Press.

Steinle, Friedrich. 1997. "Entering New Fields: Exploratory Uses of Experimentation." Philosophy of Science 64 (Supplement):S65-S74.

Steinle, Friedrich. 2002. "Experiments in History and Philosophy of Science." Perspectives on Science 10:408-432.

Taylor, Richard E. 1969. "Inelastic Electron-Proton Scattering in the Deep Continuum Region." in Proceedings of the Fourth International Symposium on Electron and Photon Interactions at High Energies (Liverpool, September 14-20), 251-260. SLAC-Pre-print: SLAC-PUB-677.

Taylor, Richard E. 2001. "The Discovery of the Point-like Structure of Matter." Philosophical Transactions of the Royal Society of London A 359:225-240.

Teller, Paul. 1995. An interpretive Introduction to Quantum Field Theory. Princeton N.J.: Princeton University Press. 
Waters, Kenneth. 2007. "The Nature and Context of Exploratory Experimentation: An Introduction to Three Case Studies of Exploratory Research." History and Philosophy of the Life Sciences 29:275-284.

Wheeler, John A. 1937. "On the Mathematical Description of Light Nuclei by the Method of Resonating Group Structure.” Physical Review 52:1107-1122.

Wiedemann, Helmut. 2007. Particle Accelerator Physics. Berlin, Heidelberger. Springer.

Zweig, George. 1964a. "An SU3 Model for Strong Interaction Symmetry and Its Breaking." CERN preprint TH-401 (January 17, 1964) and TH-412 (February 21, 1964).

Zweig, George. 1964b. “An SU3 Model for Strong Interaction Symmetry and its Breaking II." CERN Report 8419/TH.412 (February 21, 1964). Reprinted in Developments in the Quark Theory of Hadrons, A Reprint Collection, vol I: 1964-1978, edited Don B. Lichtenberg and Peter S. Rosen, 22-101. Nonamtum, Mass.:Hadronic Press, Inc. 